\title{
Counting higher genus curves with crosscaps in Calabi-Yau orientifolds
}

\author{
Vincent Bouchard \\ Mathematical Institute, University of Oxford \\ 24-29 St. Giles', Oxford OX1 3LB, U.K. \\ E-mail: bouchard@maths.ox.ac.uk

\section{Bogdan Florea} \\ Department of Physics and Astronomy, Rutgers University \\ Piscataway, NJ 08855-0849, U.S.A. \\ E-mail: florea@physics.rutgers.edu \\ Marcos Mariño* \\ Theory Division, CERN \\ Geneva 23, CH-1211, Switzerland \\ E-mail: marcos@mail.cern.ch
}

ABStRaCT: We compute all loop topological string amplitudes on orientifolds of local Calabi-Yau manifolds, by using geometric transitions involving SO/Sp Chern-Simons theory, localization on the moduli space of holomorphic maps with involution, and the topological vertex. In particular we count Klein bottles and projective planes with any number of handles in some Calabi-Yau orientifolds.

KEYwords: Chern-Simons Theories, Topological Strings.

\footnotetext{
${ }^{*}$ Also at Departamento de Matemática, IST, Lisboa, Portugal
} 


\section{Contents}

1. Introduction 1

2. A-model topological strings on an orientifold 3

2.1 Type-IIA superstrings and topological strings on an orientifold

2.2 Structure of the topological string amplitudes

3. Geometric transitions 5

3.1 Orientifold of the resolved conifold and its geometric transition

3.2 Our main example

4. Closed string amplitudes from Chern-Simons theory 9

4.1 Results from Chern-Simons theory with classical gauge groups

4.2 Computation of open string amplitudes 13

4.3 Duality map and closed string amplitudes 16

4.4 The oriented contribution

5. Unoriented localization 22

5.1 Unoriented localization @ 2 crosscaps @ degree $2 \mathbb{R P}^{2}$

5.2 Unoriented localization @ 2 crosscaps @ degree $4 \mathbb{R P}^{2}$

6. Topological vertex computation 27

6.1 General prescription 28

6.2 Examples 29

7. Discussion and open problems 30

A. Subsets of Young tableaux 31

B. Results in the general case 31

\section{Introduction}

The large- $N$ duality between open and closed topological strings, which was first formulated for local conifold transitions [1], has been extended in various directions. It has been shown to be valid for more general toric geometries, leading to the definition of the topological vertex [2], a cubic field theory that computes the all genus amplitudes of open and closed topological strings on any non-compact toric Calabi-Yau threefold. The large- $N$ duality has also been extended in [3] to a simple orientifold theory, namely the orientifold of the conifold. 
In this paper we propose a generalization of the large- $N$ correspondence of [B] for more complicated orientifolds. Namely, we find that the partition function of closed topological strings on the orientifold (including unoriented contributions and oriented contributions from the covering space) is equivalent in the large- $N$ limit to the Chern-Simons partition function on the threefold after a geometric transition. The $\mathbb{P}^{1}$ 's that were invariant under the involution, becoming $\mathbb{R P}^{2}$ 's in the orientifold, give $\mathrm{SO}(N)$ - or $\operatorname{Sp}(N)$ - Chern-Simons theory on the $\mathbf{S}^{3}$ 's resulting from the geometric transition. One has also to add instanton contributions localized on the fixed locus of a torus action on the deformed geometry.

This is a highly non-trivial proposal, as more complicated orientifolds involve instanton contributions to the Chern-Simons partition function. Moreover, for more general orientifolds, the geometry of the covering space becomes quite different from the one of the resulting orientifold. It is not obvious at all that both the oriented and unoriented contributions to the closed topological strings partition function are encoded in the Chern-Simons setup. But it turns out to be true in the examples we consider.

We also find that the closed topological string amplitudes on the orientifolds of the type we describe below can be also computed with the topological vertex introduced in [2], by using a prescription that takes into account the involution of the target. We explicitly prove that this prescription is equivalent to the large- $N$ Chern-Simons dual. This prescription extends the general formalism of the topological vertex to include the case of orientifolds.

To test our result we compute the unoriented contributions on the closed topological strings side using the unoriented localization techniques developed in 国. This computation does not rely on large- $N$ duality at all, consequently providing an independent check of our proposal. In [3] it was found that only unoriented maps with one crosscap contribute to the partition function. However, in the general case, we find that configurations with two crosscaps, that is Klein bottles, do contribute as well.

To make the proposal more concrete we focus on a particular geometry in this paper. We consider a noncompact Calabi-Yau threefold $X$ whose compact locus consists of two compact divisors each isomorphic to a del Pezzo surface $d P_{2}$ and a rational $(-1,-1)$ curve that intersects both divisors transversely. The divisors do not intersect each other. We will equip $X$ with a freely acting antiholomorphic involution $I$ and consider an orientifold of the theory obtained by gauging the discrete symmetry $\sigma I$, where $\sigma$ is a worldsheet antiholomorphic involution. The geometry is described in more detail in section 3

The partition function of the closed topological A-model with this geometry as target space will sum both over maps from orientable worldsheets to $X$ (with the Kähler parameters identified by the involution set equal) as well as over non-orientable worldsheets to the orientifolded geometry.

The orientifolded geometry allows a local geometric transition that will be described in detail in section 3. This amounts to contracting two $\mathbb{P}^{1}$ 's and an $\mathbb{R P}^{2}$ and replace them by three $\mathbf{S}^{3}$ s. We conjecture that the dual open string model will consist of a system of Chern-Simons theories supported on the three spheres, with $\mathrm{U}\left(N_{1}\right)$ and $\mathrm{U}\left(N_{2}\right)$ groups on the spheres corresponding to the contracted $\mathbb{P}^{1}$ 's and $\mathrm{SO}\left(N_{3}\right)$ - or $\operatorname{Sp}\left(N_{3}\right)$ - group on the sphere corresponding to the contracted $\mathbb{R P}^{2}$. The new ingredient is that the Chern-Simons theories will be coupled by cylindrical instantons. 
The paper is organized as follows. Section 2 summarizes general results for A-model topological strings on an orientifold. Section 3 describes in some detail the particular geometry and the geometric transition on which we will focus in this paper. In section 4 we compute explicitly the Chern-Simons amplitude obtained after the geometric transition. Section 5 presents the unoriented localization computation, and shows that it gives exactly the same contributions for the one and two crosscaps instanton configurations. We then propose our prescription based on the topological vertex in section 6, proving its equivalence to the Chern-Simons computation. Finally, in section 나, we point out some possible extensions of our results to more complicated situations.

\section{A-model topological strings on an orientifold}

\subsection{Type-IIA superstrings and topological strings on an orientifold}

It is a well-known fact that, when type-IIA theory is compactified on a Calabi-Yau manifold $X$, the resulting four dimensional theory is $\mathcal{N}=2$ supergravity with $h^{1,1}(X)$ vector multiplets $t_{i}$. The $\mathcal{N}=2$ prepotential that governs the effective action of the vector multiplets, $F_{0}\left(t_{i}\right)$, can be computed by the genus zero free energy of the A-model topological strings with the Calabi-Yau as target space (see [5] for a review of topological strings and related issues). Higher genus free energies $F_{g}\left(t_{i}\right)$ of the topological string theory also play a role in the four dimensional supergravity theory, and compute higher curvature couplings involving the graviphoton $[6,7]$.

One way to break $\mathcal{N}=2$ supersymmetry down to $\mathcal{N}=1$ is to consider an orientifold of the theory. The orientifold is defined by combining an involution symmetry $I$ on the Calabi-Yau $X$ with an orientation reversal diffeomorphism $\sigma$ on the worldsheet $\Sigma$. In the context of type-IIA superstrings, the orientifold is only well defined if the involution is anti-holomorphic. Furthermore, the worldsheet diffeomorphism has to be orientation reversal [8, [4, 9]. The resulting theory in four dimensions has $\mathcal{N}=1$ supersymmetry, and $h_{-}^{1,1}(X)$ out of the $h^{1,1}(X) \mathcal{N}=2$ vector multiplets become $\mathcal{N}=1$ chiral multiplets in four dimensions, where $h_{-}^{1,1}(X)$ is the number of harmonic $(1,1)$ forms on $X$ which have -1 eigenvalue under $I$ (see 9 for a description of the spectrum of massless modes in four dimensions).

These considerations hold in the context of A-model topological strings as well: Amodel topological strings possess a worldsheet orientation reversal symmetry when accompanied with an anti-holomorphic involution of the target space [8. It is thus possible to consider A-model topological strings on an orientifold defined as above. The twisted sector of the topological string amplitude on the orientifold includes amplitudes for unoriented Riemann surfaces. Recall that a closed, non-orientable Riemann surface is characterized by its genus $g$ and by the number of crosscaps $c$, which can be one or two (crosscaps can be traded for handles when the number of crosscaps is higher than two). For example, the surface with $g=0$ and $c=1$ is the real projective plane $\mathbb{R P}^{2}$, while the surface with $g=0$ and $c=2$ is the Klein bottle. It was shown in [8] that the superpotential of the $h_{-}^{1,1}(X)$ chiral multiplets is given by the $\mathbb{R P}^{2}$ amplitude of the topological string theory. As far as 
we know, the topological amplitudes involving more handles or crosscaps do not have an interpretation in the $\mathcal{N}=1$ supergravity theory.

Generally speaking, one could consider type-IIA superstrings on a non-compact orientifold, with D-branes and orientifold planes [8]. In this paper we will only consider type-IIA superstrings without D-branes or orientifold planes (albeit our approach could probably be generalized to these cases). This means that the anti-holomorphic involution must have no fixed points. Moreover, as the parent theory has no D-branes, to compute the superpotential we only need to consider A-model closed topological strings.

\subsection{Structure of the topological string amplitudes}

Roughly speaking, the free energy of A-model closed topological strings counts the number of holomorphic maps from the worldsheet to the target space, weighted by a factor of $e^{-A}$ where $A$ is the area of the embedded curve. In the context of orientifolds, the partition function of topological strings sums over holomorphic maps in two different sectors: the "untwisted" and the "twisted" sectors. The former consists of usual holomorphic maps from orientable worldsheets to the covering space, i.e. the non-compact Calabi-Yau threefold without the involution. The latter consists of equivariant maps $f: \Sigma \rightarrow X$ satisfying the equivariance condition

$$
f \circ \sigma=I \circ f,
$$

where $I$ is the antiholomorphic involution acting on $X$, and $\sigma: \Sigma \rightarrow \Sigma$ is the orientation reversal diffeomorphism of the Riemann surface which is needed in order to construct the orientifold action. Notice that, if $\Sigma$ has genus zero, the action of $\sigma$ is given by $z \rightarrow-1 / \bar{z}$. The relevant maps in the twisted sector are then the maps which are compatible with the orientation reversal diffeomorphism on the worldsheet and the anti-holomorphic involution on the target space, and descend to holomorphic maps from non-orientable worldsheets to the orientifold.

The structure of the total free energy of the $\mathbf{A}$-model is then

$$
\mathcal{F}\left(\frac{X}{I}, g_{s}\right)=\mathcal{F}\left(\frac{X}{I}, g_{s}\right)_{\text {or }}+\mathcal{F}\left(\frac{X}{I}, g_{s}\right)_{\text {unor }},
$$

where $g_{s}$ is the string coupling constant. In this equation, $\mathcal{F}\left(X / I, g_{s}\right)_{\text {or }}$ is the contribution of the untwisted sector, and $\mathcal{F}\left(X / I, g_{s}\right)$ unor is the contribution of the twisted sector. Moreover, we have [10, 3]

$$
\mathcal{F}\left(\frac{X}{I}, g_{s}\right)_{\text {or }}=\frac{1}{2} \mathcal{F}\left(X, g_{s}\right)=\frac{1}{2} \sum_{d=1}^{\infty} \sum_{g=0}^{\infty} \sum_{Q} \frac{1}{d} \frac{n_{Q}^{g}}{\left(q^{\frac{d}{2}}-q^{-\frac{d}{2}}\right)^{2-2 g}} e^{-d Q \cdot t} .
$$

Here, $\mathcal{F}\left(X, g_{s}\right)$ is the free energy of the covering $X$ of $X / I$, after suitably identifying the Kähler classes in the way prescribed by the involution $I$, and we have written it in terms of Gopakumar-Vafa invariants $n_{Q}^{g}[10]$. The notation is as follows: $t=\left(t_{1}, \ldots, t_{n}\right)$ denotes the set of Kähler parameters of $X$ after identification through the involution, $Q=\left(Q_{1}, \ldots, Q_{n}\right)$ is an $n$-uple of integer numbers that label integer two-homology classes, and $q=e^{g_{s}}$. 
The unoriented contribution in (2.2) comes from holomorphic maps from closed nonorientable Riemann surfaces to the orientifold $X / I$. The Euler characteristic of a closed Riemann surface of genus $g$ and $c$ crosscaps is $\chi=-2 g+2-c$ where $c$ is the number of crosscaps. We then have

$$
\mathcal{F}\left(\frac{X}{I}, g_{s}\right)_{\text {unor }}=\mathcal{F}\left(\frac{X}{I}, g_{s}\right)_{\text {unor }}^{c=1}+\mathcal{F}\left(\frac{X}{I}, g_{s}\right)_{\text {unor }}^{c=2}
$$

which corresponds to the contributions of one and two crosscaps. Following the arguments in 10 we expect the structure

$$
\begin{aligned}
& \mathcal{F}\left(\frac{X}{I}, g_{s}\right)_{\text {unor }}^{c=1}= \pm \sum_{d \text { odd } g=0} \sum_{Q}^{\infty} \sum_{Q} n_{Q}^{g, c=1} \frac{1}{d}\left(q^{\frac{d}{2}}-q^{-\frac{d}{2}}\right)^{2 g-1} e^{-d Q \cdot t}, \\
& \mathcal{F}\left(\frac{X}{I}, g_{s}\right)_{\text {unor }}^{c=2}=\sum_{d \text { odd }} \sum_{g=0}^{\infty} \sum_{Q} n_{Q}^{g, c=2} \frac{1}{d}\left(q^{\frac{d}{2}}-q^{-\frac{d}{2}}\right)^{2 g} e^{-d Q \cdot t}
\end{aligned}
$$

where $n_{Q}^{g, c}$ are integers. The \pm sign in the $c=1$ free energy is due to the following: the target space anti-holomorphic involution does not fully specify the unoriented part of the free energy on the orientifold, since we have to make a choice for the sign of the crosscaps. Depending on this choice, we will have the two different signs for $c=1$. This corresponds to the choice of $S O$ or $S p$ group in the gauge theory dual. This remaining choice is also easily understood on the mirror symmetric side [B]. For the conifold, the B-model mirror symmetric description involves two orientifold 5-planes. The two choices of signs for crosscap states correspond on the mirror symmetric side to the two following choices for the charges of the O5-planes: +- and $-+[\mathbb{B}]$. A similar story holds for more complicated orientifolds. Notice as well that the sum over multicoverings $d$ in (2.5) is only over odd integers. In the case of $c=1$ this follows from an elementary geometric argument, since there are no even multicoverings (see [3, 8). For $c=2$ there is no such a simple argument, but our explicit computations both in Chern-Simons theory and in localization of unoriented instantons indicate that only odd multicoverings contribute.

\section{Geometric transitions}

\subsection{Orientifold of the resolved conifold and its geometric transition}

In [3] it was proposed that in the large- $N$ limit, closed topological strings on the orientifold of the conifold are dual to $\mathrm{SO}(N) / \mathrm{Sp}(N)$ Chern-Simons theory on $\mathbf{S}^{3}$, where the choice of gauge group is related to the choice of sign for the crosscaps. Since this is the starting point for our discussion, let us review in some detail the results of [3.

We start with a theory of topological open strings on the deformed conifold defined by $z_{1} z_{4}-z_{2} z_{3}=\mu$. The conifold contains an $\mathbf{S}^{3}$, and if we wrap $2 N$ branes on the three-sphere, the spacetime description of the open topological string theory is Chern-Simons theory on $\mathbf{S}^{3}$ with gauge group $\mathrm{U}(2 N)$ and at level $k$ (the level is related to the open string coupling constant). We now consider the following involution of the geometry

$$
I: \quad\left(z_{1}, z_{2}, z_{3}, z_{4}\right) \rightarrow\left(\bar{z}_{4},-\bar{z}_{3},-\bar{z}_{2}, \bar{z}_{1}\right)
$$


that leaves the $\mathbf{S}^{3}$ invariant. The string field theory for the resulting open strings is now Chern-Simons theory with gauge group $\mathrm{SO}(N)$ or $\mathrm{Sp}(N)$, depending on the choice of orientifold action on the gauge group. The total free energy of the Chern-Simons theory with gauge group SO / Sp can be written as

$$
\mathcal{F}=-\log S_{00}^{\mathrm{SO}(N) / \mathrm{Sp}(N)}=\frac{1}{2} \sum_{d=1}^{\infty} \frac{1}{d} \frac{e^{-d t}}{\left(q^{\frac{d}{2}}-q^{-\frac{d}{2}}\right)^{2}} \mp \sum_{d \text { odd }} \frac{1}{d} \frac{e^{-d t / 2}}{q^{\frac{d}{2}}-q^{-\frac{d}{2}}},
$$

where the $\mp$ sign corresponds to $\mathrm{SO} / \mathrm{Sp}$, respectively. In (3.2),$q=e^{g_{s}}$, with

$$
g_{s}=\frac{2 \pi i}{k+y},
$$

and $y$ is the dual Coxeter of the gauge group, which is $N-2$ for $\mathrm{SO}(N)$ and $N+1$ for $\operatorname{Sp}(N)$. The parameter $t$ in (3.2) is the 't Hooft parameter, given by

$$
t=(N \mp 1) g_{s}
$$

for $\mathrm{SO} / \mathrm{Sp}$, respectively.

In the usual geometric transition of [1] , the dual to the deformed conifold is the resolved conifold $Y=\mathcal{O}(-1) \oplus \mathcal{O}(-1) \rightarrow \mathbb{P}^{1}$. This Calabi-Yau threefold admits a toric description given by the following toric data:

$$
\begin{array}{ccccc} 
& X_{1} & X_{2} & X_{3} & X_{4} \\
\mathbb{C}^{*} & 1 & 1 & -1 & -1 .
\end{array}
$$

This means that $Y$ is defined as the space obtained from

$$
\left|X_{1}\right|^{2}+\left|X_{2}\right|^{2}-\left|X_{3}\right|^{2}-\left|X_{4}\right|^{2}=t
$$

after quotienting by the $\mathrm{U}(1)$ action specified by the charges in (3.5). The involution (3.1) of the deformed conifold maps to the antiholomorphic involution of $Y$ defined by:

$$
I: \quad\left(X_{1}, X_{2}, X_{3}, X_{4}\right) \rightarrow\left(\bar{X}_{2},-\bar{X}_{1}, \bar{X}_{4},-\bar{X}_{3}\right) .
$$

It is easy to see that $Y / I$ contains a single $\mathbb{R P}^{2}$ obtained from the quotient of the $\mathbb{P}^{1}$ of $Y$ by $I$. We will represent the quotient of the resolved conifold by that involution in terms of the toric diagram depicted in figure 1 .

The free energy of the SO / Sp Chern-Simons theory gives the total free energy of closed strings propagating on $Y / I$. The first term in (3.2) gives the oriented contribution, while the second term gives the unoriented contribution, and they have the structure explained in (2.3) and (2.5). Notice that in the case of the unoriented contribution we have

$$
n_{Q=1 / 2}^{g=0, c=1}=\mp 1
$$

depending on the choice of sign for the crosscaps, and all the remaining Gopakumar-Vafa invariants vanish. In particular, the contribution of Riemann surfaces with two crosscaps is zero. As we will see in this paper, in more general cases there are two-crosscaps contributions. The above prediction of the large- $N$ transition for the free energy was checked in [8] against mirror symmetry, and in [-] against localization computations for unoriented Gromov-Witten theory. 


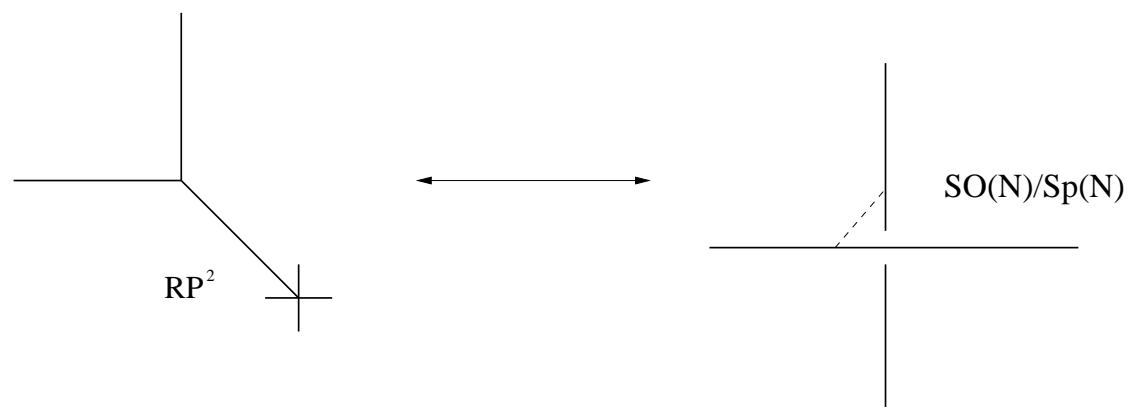

Figure 1: Geometric transition for the orientifold of the conifold. The cross in the figure to the left represents an $\mathbb{R P}^{2}$ obtained by quotienting a $\mathbb{P}^{1}$ by the involution $I$, and the dashed line in the figure on the right represents an $\mathbf{S}^{3}$ with $\mathrm{SO} / \mathrm{Sp}$ gauge group.

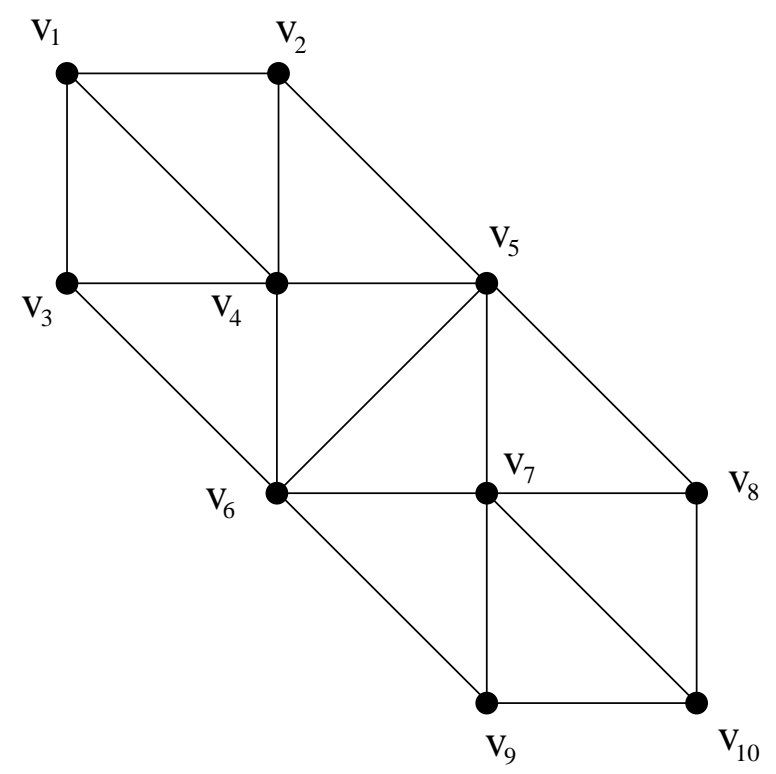

Figure 2: Toric diagram of the noncompact Calabi-Yau threefold $X$.

\subsection{Our main example}

In this paper we want to generalize the open/closed string duality studied in [3] to more general orientifolds. We will mainly focus on a noncompact Calabi-Yau manifold $X$ described by the following toric data:

$\begin{array}{lcccccccccc} & X_{1} & X_{2} & X_{3} & X_{4} & X_{5} & X_{6} & X_{7} & X_{8} & X_{9} & X_{10} \\ \mathbb{C}^{*} & -1 & 1 & 1 & -1 & 0 & 0 & 0 & 0 & 0 & 0 \\ \mathbb{C}^{*} & 1 & 0 & -1 & -1 & 0 & 1 & 0 & 0 & 0 & 0 \\ \mathbb{C}^{*} & 1 & -1 & 0 & -1 & 1 & 0 & 0 & 0 & 0 & 0 \\ \mathbb{C}^{*} & 0 & 0 & 0 & 1 & -1 & -1 & 1 & 0 & 0 & 0 \\ \mathbb{C}^{*} & 0 & 0 & 0 & 0 & 1 & 0 & -1 & -1 & 0 & 1 \\ \mathbb{C}^{*} & 0 & 0 & 0 & 0 & 0 & 1 & -1 & 0 & -1 & 1 \\ \mathbb{C}^{*} & 0 & 0 & 0 & 0 & 0 & 0 & -1 & 1 & 1 & -1 .\end{array}$




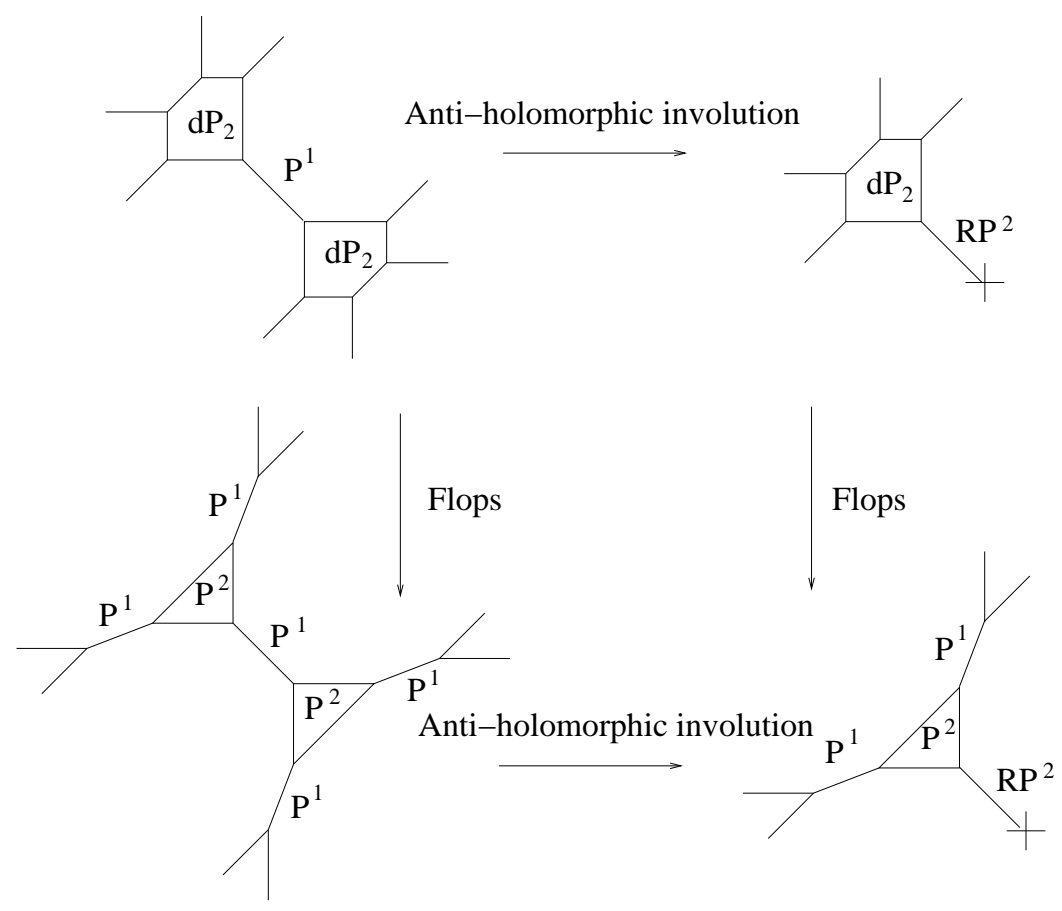

Figure 3: The geometry on the closed topological strings side. The orientifolding action acts from left to right, while flopping the $\mathbb{P}^{1}$ 's acts top-down. The $\mathbb{R P}^{2}$ is represented by a cross at the end of the toric leg.

The compact locus consists of two divisors that are each isomorphic to a del Pezzo surface $d P_{2}$ and a rational $(-1,-1)$ curve that intersects both divisors transversely. Note that the two compact divisors do not intersection We now consider a real torus action on $X$ given by:

$$
e^{i \phi} \cdot\left(X_{1}, X_{2}, \ldots, X_{10}\right) \rightarrow\left(e^{i \lambda_{1} \phi} X_{1}, e^{i \lambda_{2} \phi} X_{2}, \ldots, e^{i \lambda_{10} \phi} X_{10}\right)
$$

The configuration of invariant curves is presented in figure 3. We now define the antiholomorphic involution as follows:

$$
\begin{aligned}
I: \quad & \left(X_{1}, X_{2}, X_{3}, X_{4}, X_{5}, X_{6}, X_{7}, X_{8}, X_{9}, X_{10}\right) \rightarrow \\
\quad & \rightarrow\left(\bar{X}_{10}, \bar{X}_{8}, \bar{X}_{9}, \bar{X}_{7},-\bar{X}_{6}, \bar{X}_{5},-\bar{X}_{4}, \bar{X}_{2}, \bar{X}_{3}, \bar{X}_{1}\right) .
\end{aligned}
$$

The subtorus of (3.10) that is compatible with the involution is defined by the following constraints on the weigths

$$
\lambda_{1}+\lambda_{10}=0, \quad \lambda_{2}+\lambda_{8}=0, \quad \lambda_{3}+\lambda_{9}=0, \quad \lambda_{4}+\lambda_{7}=0, \quad \lambda_{5}+\lambda_{6}=0 .
$$

Imposing these constraints does not enlarge the set of invariant curves.

It is often useful [1] to consider a related Calabi-Yau threefold $\widetilde{X}$ obtained from $X$ by flopping the two exceptional curves outside of the compact divisors. The "commuting square" of geometries (where the arrows correspond either to flopping or to quotienting by the antiholomorphic involution) is presented in figure 3. 


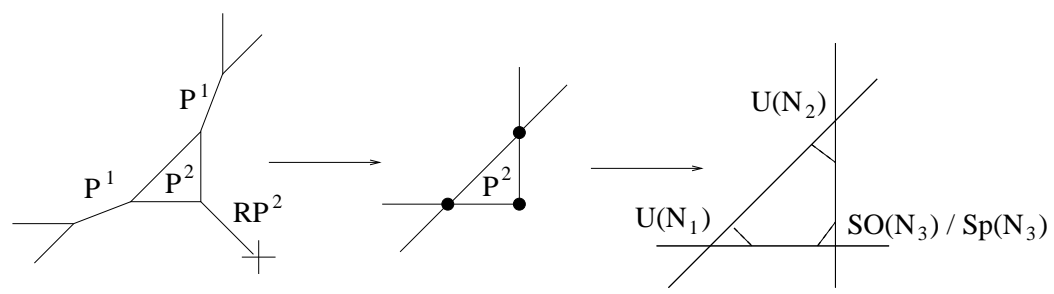

Figure 4: The geometric transition. The two $\mathbb{P}^{1}$ and the $\mathbb{R P}^{2}$ of the left figure are shrunk to singular points in the middle diagram, and then deformed into three $S^{3}$.

We can now follow the logic in [12, 13, 14, 11] and consider a geometric transition in which each of the resolved conifolds (or their orientifolds) that exist locally in the geometry are replaced by deformed conifolds (or their orientifolds). In the above example, this means that we contract two $\mathbb{P}^{1}$ 's and an $\mathbb{R P}^{2}$ and we replace them with three spheres carrying $\mathrm{U}(N)$ and $\mathrm{SO}(N) / \mathrm{Sp}(N)$ Chern-Simons theories, respectively. The transition is represented in figure 3. In the next section we will see how to obtain the closed string amplitudes in the orientifold from Chern-Simons theory

\section{Closed string amplitudes from Chern-Simons theory}

\subsection{Results from Chern-Simons theory with classical gauge groups}

As we will see in a moment, in order to compute the free energies of topological strings on orientifolds via geometric transitions we have to compute the Chern-Simons invariants of the unknot and the Hopf link of linking number +1 in arbitrary representations of $\mathrm{U}(N)$, $\mathrm{SO}(N)$ and $\operatorname{Sp}(N)$. In this section we will denote $q=e^{g_{s}}$ and

$$
\lambda=q^{N+a},
$$

where

$$
a= \begin{cases}0 & \text { for } \mathrm{U}(N), \\ -1 & \text { for } \mathrm{SO}(N), \\ 1 & \text { for } \operatorname{Sp}(N) .\end{cases}
$$

Notice that the 't Hooft parameter of the classical gauge groups can be written as

$$
t=(N+a) g_{s}
$$

therefore $\lambda=e^{t}$. For an arbitrary gauge group $G$ it is a well known result that the ChernSimons invariant of the unknot in an arbitrary representation $R$ is given by the so-called quantum dimension of $R$ [15]:

$$
\mathcal{W}_{R}=\frac{S_{0 R}}{S_{00}}=\operatorname{dim}_{q} R
$$

where $S_{0 R}, S_{00}$ are entries of the $S$ matrix of the WZW model with the corresponding gauge group and at level $k$ (recall that $k$ is related to the string coupling constant by (3.3)). 
Using Weyl's formula one can write the quantum dimension as a product over positive roots $\alpha \in \Delta_{+}$:

$$
\operatorname{dim}_{q} R=\prod_{\alpha \in \Delta_{+}} \frac{\left[\left(\Lambda_{R}+\rho, \alpha\right)\right]}{[(\rho, \alpha)]}
$$

where $\Lambda_{R}$ is the highest weight of the representation $R$ and $\rho$ is the Weyl vector. We also defined the following $q$-number:

$$
[x]=q^{x / 2}-q^{-x / 2} .
$$

The expression (4.5) can be written more explicitly for the different classical gauge groups. Let $R$ be a representation corresponding to a Young tableau with row lengths $\left\{\mu_{i}\right\}_{i=1, \ldots, d(\mu)}$, with $\mu_{1} \leq \mu_{2} \leq \cdots$ and where $d(\mu)$ denotes the number of rows. Then the quantum dimension of a representation $R$ of $\mathrm{U}(N)$ reads (see for example [16])

$$
\operatorname{dim}_{q}^{\mathrm{U}(N)} R=\prod_{1 \leq i<j \leq d(\mu)} \frac{\left[\mu_{i}-\mu_{j}+j-i\right]}{[j-i]} \prod_{i=1}^{d(\mu)} \frac{\prod_{v=-i+1}^{\mu_{i}-1}[v]_{\lambda}}{\prod_{w=1}^{\mu_{i}}[w-i+d(\mu)]},
$$

where we defined

$$
[x]_{\lambda}=\lambda^{1 / 2} q^{x / 2}-\lambda^{-1 / 2} q^{-x / 2},
$$

and $\lambda=q^{N}$ for $\mathrm{U}(N)$ representations.

We can also find explicit expressions for the quantum dimensions of $\mathrm{SO}(N)$ and $\operatorname{Sp}(N)$ representations

$$
\begin{aligned}
\operatorname{dim}_{q}^{\mathrm{SO}(N)} R= & \prod_{1 \leq i<j \leq d(\mu)} \frac{\left[\mu_{i}-\mu_{j}+j-i\right]\left[\mu_{i}+\mu_{j}+1-i-j\right]_{\lambda}}{[j-i][1-i-j]_{\lambda}} \times \\
& \times \prod_{i=1}^{d(\mu)} \frac{\left[\mu_{i}-i\right]_{\lambda}^{\mathrm{SO}(N)}}{[-i]_{\lambda}^{\mathrm{SO}(N)}} \prod_{v=1}^{\mu_{i}} \frac{\left[\mu_{i}+1-i-v-d(\mu)\right]_{\lambda}}{[v-i+d(\mu)]}, \\
\operatorname{dim}_{q}^{\mathrm{Sp}(N)} R= & \prod_{1 \leq i<j \leq d(\mu)} \frac{\left[\mu_{i}-\mu_{j}+j-i\right]\left[\mu_{i}+\mu_{j}+1-i-j\right]_{\lambda}}{[j-i][1-i-j]_{\lambda}} \times \\
& \times \prod_{i=1}^{d(\mu)} \frac{[1-i]_{\lambda}^{\mathrm{Sp}(N)}\left[2 \mu_{i}-2 i+1\right]_{\lambda}}{\left[1-i+\mu_{i}\right]_{\lambda}^{\operatorname{Sp}(N)}[1-2 i]_{\lambda}} \prod_{v=1}^{\mu_{i}} \frac{\left[\mu_{i}+1-i-v-d(\mu)\right]_{\lambda}}{[v-i+d(\mu)]},
\end{aligned}
$$

where we defined

$$
\begin{aligned}
& {[x]_{\lambda}^{\mathrm{SO}(N)}=\lambda^{1 / 4} q^{\frac{1}{4}(2 x+1)}-\lambda^{-1 / 4} q^{-\frac{1}{4}(2 x+1)},} \\
& {[x]_{\lambda}^{\operatorname{Sp}(N)}=\lambda^{1 / 4} q^{\frac{1}{4}(2 x-1)}-\lambda^{-1 / 4} q^{-\frac{1}{4}(2 x-1)},}
\end{aligned}
$$

with $\lambda=q^{N+a}$ which leads to $\lambda=q^{N-1}$ for $\operatorname{SO}(N)$ and $\lambda=q^{N+1}$ for $\operatorname{Sp}(N)$. Using (4.9) one can show that

$$
\operatorname{dim}_{q}^{\mathrm{Sp}(N)} R=(-1)^{\ell(R)} \operatorname{dim}_{q}^{\mathrm{SO}(-N)} R^{T}
$$

where $R^{T}$ is the transposed or conjugate representation, related to $R$ by exchanging rows with columns, $\mathrm{SO}(-N)$ is meant in the sense of analytic continuation, and $\ell(R)$ is the 
number of boxes of the Young tableau. This relation is part of the " $\mathrm{SO}(N)=\operatorname{Sp}(-N)$ " equivalence [17]. A relation similar to (4.11) holds for usual dimensions [18].

Using (4.7) and (4.9) one can also infer the following formula for quantum dimensions of representations of $\mathrm{SO}(N)$ and $\mathrm{Sp}(N)$ in terms of quantum dimensions of representations of $\mathrm{U}(N)$ :

$$
\operatorname{dim}_{q}^{\mathrm{SO}(N) / \mathrm{Sp}(N)} R=\sum_{Q=Q^{t}}(-1)^{1 / 2(\ell(Q) \mp r(Q))} \operatorname{dim}_{q}^{\mathrm{U}(N)}\left(\frac{R}{Q}\right),
$$

where the skew quantum dimension is defined by

$$
\operatorname{dim}_{q}^{\mathrm{U}(N)}(R / Q)=\sum_{R^{\prime}} N_{R^{\prime} Q}^{R} \operatorname{dim}_{q}^{\mathrm{U}(N)} R^{\prime}
$$

and $N_{R_{1} R_{2}}^{R}$ are the usual Littlewood-Richardson coefficients that appear in the tensor product of $\mathrm{U}(N)$ representations: $R_{1} \otimes R_{2}=\sum_{R} N_{R_{1} R_{2}}^{R} R$. In (4.12) the sum is over self-conjugate representations, i.e. representations that are equal to their transpose, and starts with the trivial representation: $\{\cdot, \square, \boxplus, \boxplus, \boxplus, \ldots\} . r(Q)$ denotes the rank of $Q$, which is defined as the number of boxes in the leading diagonal of the Young tableau [19]. The - sign is for $\operatorname{SO}(N)$ representations while the $+\operatorname{sign}$ is for $\operatorname{Sp}(N)$ representations.

As we will see in the following sections, the relations between quantum dimensions of representations of $\mathrm{SO}(N)$ and $\mathrm{Sp}(N)$ (4.11) and (4.12) are responsible for the fact that partition functions of $\mathrm{SO}(N)$ and $\operatorname{Sp}(N)$ differ only by an overall sign in front of the unoriented contributions with an odd number of crosscaps, which lead to the interpretation that they correspond to different choices of sign for the crosscap states. Basically, the first term in the sum of the right hand side of $(4.12)$ is responsible for oriented contributions to the partition functions, so they are the same for $\mathrm{SO}(N), \operatorname{Sp}(N)$ and $\mathrm{U}(N)$ gauge groups. The other terms in the sum are responsible for unoriented contributions to the partition function, and the difference of sign in the exponent of the $(-1)$ factor leads to a relative minus sign between unoriented contributions with an odd number of crosscaps of the $\mathrm{SO}(N)$ and $\operatorname{Sp}(N)$ partition functions.

Another important ingredient we will need is the framing of knots and links [15]. Given a knot invariant in representation $R$, we can change its framing by $p$ units (where $p$ is an integer) if we multiply it by

$$
(-1)^{\ell(R) p} q^{p C_{R} / 2}
$$

where $C_{R}$ is the quadratic Casimir of the representation $R$. The quadratic Casimirs have the following expressions for the different classical gauge groups:

$$
C_{R}=\kappa_{R}+(N+a) \ell(R)
$$

where $a$ is given by (4.2), and

$$
\kappa_{R}=\sum_{i} \mu_{i}\left(\mu_{i}-2 i+1\right)
$$

The framing factor can then be written as

$$
(-1)^{\ell(R) p} \lambda^{p \ell(R) / 2} q^{p \kappa_{R} / 2} .
$$


The sign in (4.14) is not standard in the context of Chern-Simons theory, but as shown in [20, 16], it is crucial in the context of topological string theory in order to guarantee integrality properties in the resulting amplitudes. To incorporate a change of framing in a link, we just change the framings of each of its components according to the rule (4.14) as well.

In our computations we will also need the invariants of Hopf links with linking number +1 . For arbitrary gauge group $\mathcal{G}$, the invariant of the Hopf link with linking number +1 is given by the normalized inverse $S$ matrix [15], and it can be written in terms of quantum dimensions as (see for example [21])

$$
\mathcal{W}_{R_{1} R_{2}}=\frac{S_{R_{1} R_{2}}^{-1}}{S_{00}}=\sum_{R \in R_{1} \otimes R_{2}} q^{\frac{1}{2}\left(C_{R}-C_{R_{1}}-C_{R_{2}}\right)} \operatorname{dim}_{q} R,
$$

where the sum is over all representations $R$ occurring in the decomposition of the tensor product of $R_{1}$ and $R_{2}$. In the $\mathrm{U}(N)$ case, we can replace the Casimir operators $C_{R_{i}}$ appearing in (4.18) by $\kappa_{R_{i}}$, since $\ell(R)=\ell\left(R_{1}\right)+\ell\left(R_{2}\right)$ in the decomposition of a tensor product of irreducible representations of $\mathrm{U}(N)$. However this relation between the number of boxes of Young tableaux does not hold in the $\operatorname{SO}(N)$ and $\operatorname{Sp}(N)$ cases. We thus find

$$
\begin{aligned}
\mathcal{W}_{R_{1} R_{2}}^{\mathrm{U}(N)} & =\sum_{R} N_{R_{1} R_{2}}^{R} q^{\frac{1}{2}\left(\kappa_{R}-\kappa_{R_{1}}-\kappa_{R_{2}}\right)} \operatorname{dim}_{q}^{\mathrm{U}(N)} R, \\
\mathcal{W}_{R_{1} R_{2}}^{\mathrm{SO}(N) / \mathrm{Sp}(N)} & =\sum_{R} M_{R_{1} R_{2}}^{R} \lambda^{\frac{1}{2}\left(\ell(R)-\ell\left(R_{1}\right)-\ell\left(R_{2}\right)\right)} q^{\frac{1}{2}\left(\kappa_{R}-\kappa_{R_{1}}-\kappa_{R_{2}}\right)} \operatorname{dim}_{q}^{\mathrm{SO}(N) / \mathrm{Sp}(N)} R,
\end{aligned}
$$

where we have denoted by $M_{R_{1} R_{2}}^{R}$ the tensor product coefficients for irreducible representations of $\mathrm{SO}(N)$ and $\operatorname{Sp}(N)$, which turn out to be the same for $S O$ and $S p$.

To compute (4.19) we need the values of $M_{R_{1} R_{2}}^{R}$, in other words, we have to decompose any tensor product of $\mathrm{SO}(N)$ or $\mathrm{Sp}(N)$ representations into a sum of irreducible representations. This can be done with a technique first developped by Littlewood in [19]. Let us first consider $\mathrm{SO}(N)$ representations. Let $[R]$ be the character of the representations $R$, as a function of the eigenvalues of an $\mathrm{SO}(N)$ matrix, and let $\{R\}$ be the Schur function of these eigenvalues labeled by the same representation. One can prove the following formulae [19]:

$$
\begin{aligned}
& {[R]=\{R\}+\sum_{R_{1} \in\{\delta\}}(-1)^{\ell\left(R_{1}\right) / 2} N_{R_{1} R_{2}}^{R}\left\{R_{2}\right\},} \\
& \{R\}=[R]+\sum_{R_{1} \in\{\gamma\}} N_{R_{1} R_{2}}^{R}\left[R_{2}\right],
\end{aligned}
$$

where $\{\delta\}$ and $\{\gamma\}$ are subsets of Young tableaux that we describe in appendix A. By using these relations one can express each character $[R]\left[R^{\prime}\right]$ in the product as a sum of Schur functions, then multiply these with the usual Littlewood-Richardson coefficients, and finally rexpress the Schur functions in terms of a sum of characters by the second equation of 4.20). For example,

$$
\begin{aligned}
& \text { [ロ] [ם] }=(-1+\{\square\})(\{\square\}) \\
& =\{\square\}+\{\Phi\}-\{\square\} \\
& =[\square]+[\square]+[\boxminus]+[\square]-[\square]=[\square]+[س]+[\boxplus] \text {, }
\end{aligned}
$$


where the Young tableaux are associated to irreducible representations of $\mathrm{SO}(N)$. To compute the decompositions for $\operatorname{Sp}(N)$ representations, one only has to replace the subsets $\{\delta\}$ and $\{\gamma\}$ respectively by the subsets $\{\beta\}$ and $\{\alpha\}$, which are also explained in appendix A [18]. Using this technique one can decompose any tensor products of $\mathrm{SO}(N)$ and $\mathrm{Sp}(N)$ representations into a sum of irreducible representations, which is needed in the computation of expectation values of Hopf links using (4.19). One finds that the decomposition of tensor products is always the same for $\mathrm{SO}(N)$ and $\mathrm{Sp}(N)$ representations, justifying our claim above.

The procedure we have described turns out to be rather involved, and fortunately there is a more direct way of computing $M_{R_{1} R_{2}}^{R}$ through the following formula [22, 23]:

$$
M_{R_{1} R_{2}}^{R}=\sum_{Q, T, U} N_{Q T}^{R_{1}} N_{Q U}^{R_{2}} N_{T U}^{R}
$$

which expresses these coefficients in terms of usual Littlewood-Richardson coefficients. This formula allows to easily compute the invariants of Hopf links for SO / Sp gauge groups for any pair of representations.

As shown in [1], 2], the Hopf link invariant $\mathcal{W}_{R_{1} R_{2}}^{\mathrm{U}(N)}$ plays a crucial role in the computation of oriented string amplitudes. It is a Laurent polynomial in $\lambda^{\frac{1}{2}}$ whose highest power is $\lambda^{\left(\ell\left(R_{1}\right)+\ell\left(R_{2}\right)\right) / 2}$ :

$$
\mathcal{W}_{R_{1} R_{2}}^{\mathrm{U}(N)}=\lambda^{\left(\ell\left(R_{1}\right)+\ell\left(R_{2}\right)\right) / 2} W_{R_{1} R_{2}}(q)+\cdots,
$$

where the dots refer to terms with lower powers of $\lambda$. The leading part of $\mathcal{W}_{R_{1} R_{2}}^{\mathrm{U}(N)}$, which we have denoted by $W_{R_{1} R_{2}}$, can be computed in terms of Schur polynomials in an infinite number of variables (see for example 12, 24, 25 for more details):

$$
W_{R_{1} R_{2}}(q)=s_{R_{2}}\left(x_{i}=q^{-i+\frac{1}{2}}\right) s_{R_{1}}\left(x_{i}=q^{\mu_{i}^{R_{2}}-i+\frac{1}{2}}\right),
$$

where $\left\{\mu_{i}^{R_{2}}\right\}_{i=1, \ldots, d\left(\mu^{R_{2}}\right)}$ is the partition corresponding to $R_{2}$. We will also denote $W_{R}=$ $W_{R}=s_{R}\left(x_{i}=q^{-i+\frac{1}{2}}\right)$. By looking at the formula in $(4.19)$ for $\mathcal{W}_{R_{1} R_{2}}^{\mathrm{SO}(N) / \mathrm{Sp}(N)}$, one can see that it is a Laurent polynomial in $\lambda^{\frac{1}{2}}$, whose highest power is also $\lambda^{\left(\ell\left(R_{1}\right)+\ell\left(R_{2}\right)\right) / 2}$, and which has the same leading coefficient $W_{R_{1} R_{2}}(q)$.

\subsection{Computation of open string amplitudes}

We will now follow the results in [11, 13, 14] to compute the topological open string amplitudes in the geometry described in section 3 , which is shown in figure 5 . There are $N_{i}$ D-branes wrapped around the three $\mathbf{S}^{3}$ 's $M_{i}, i=1,2,3$. This geometry is similar to the one considered for example in [11]; the main difference being that one of the spheres in our geometry, more precisely $M_{3}$, is left invariant by the anti-holomorphic involution, thus leading to a $\mathrm{SO}(N)$ or $\mathrm{Sp}(N)$ Chern-Simons theory.

For open strings with both ends on the same $\mathbf{S}^{3}$, the dynamics is described by a ChernSimons theory as usual. For $M_{1}$ and $M_{2}$, the Chern-Simons theories respectively have gauge groups $\mathrm{U}\left(N_{1}\right)$ and $\mathrm{U}\left(N_{2}\right)$, while for $M_{3}$ it has gauge group $\mathrm{SO}\left(N_{3}\right)$ or $\mathrm{Sp}\left(N_{3}\right)$. However, 


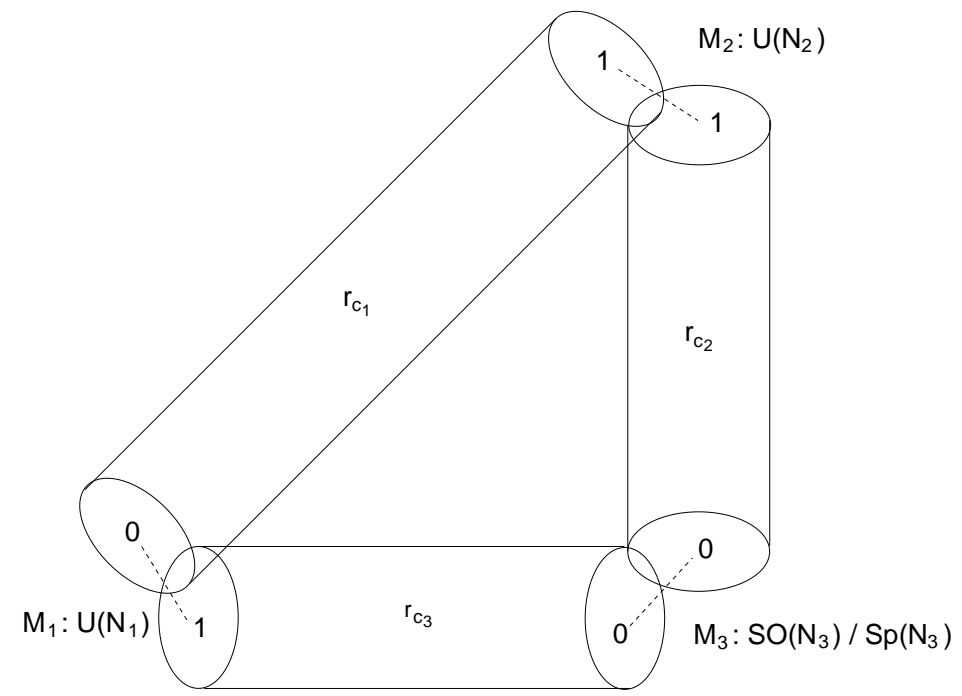

Figure 5: The deformed geometry. $M_{i} i=1,2,3$ are the three spheres and $r_{c_{i}}$ are the Kähler parameters of the cylindrical instantons. The gauge groups of the Chern-Simons theories on the spheres and the framings of the unknots are also indicated.

there are also cylindrical open string instantons coupling the Chern-Simons theories on different spheres [26]. Schematically, the path integral becomes

$$
Z=\int \prod_{i=1}^{3} \mathcal{D} A_{i} e^{\sum_{i=1}^{3} S_{i}^{C S}\left(A_{i}\right)+S_{\mathrm{inst}}}
$$

where $S_{i}^{C S}\left(A_{i}\right), i=1,2,3$ are the Chern-Simons actions for the three $\mathbf{S}^{3}$ 's. The instanton sector, $S_{\text {inst }}$, can be computed by using localization (as in [13, 14]) or by using the techniques of 111. We will follow here the procedure of [11. As explained there, the bifundamental strings stretching between two three sphere $\mathbf{S}^{3}$ 's give a massive complex scalar field, with mass proportional to the complexified Kähler parameter $r_{c}$ corresponding to the "distance" between the two spheres. After integrating out this scalar field one finds an operator which corresponds to a primitive annulus of size $r_{c}$ together with its multicovers. The boundaries of the annulus are on the two three spheres between which the bifundamental strings are stretched. These cylindrical instantons and the geometry are shown in figure 5. Inserting one operator for each cylindrical instanton we find

$$
e^{S_{\text {inst }}}=\mathcal{O}\left(U_{3}, U_{1}\right) \mathcal{O}\left(V_{1}, V_{2}\right) \mathcal{O}\left(U_{2}, V_{3}\right),
$$

where we have defined the holonomy variables

$$
U_{i}=P \exp \oint_{\Xi_{i}} A_{i}, \quad V_{i}=P \exp \oint_{\Gamma_{i}} A_{i}, \quad i=1,2,3,
$$

and the $\Xi_{i}, \Gamma_{i}, i=1,2,3$ are the boundary components of the cylindrical instantons, which are unknots in the corresponding three-spheres. The operators in (4.26) are given by

$$
\mathcal{O}\left(A, B, r_{c}\right)=\sum_{R} \operatorname{Tr}_{R} A e^{-\ell(R) r_{c}} \operatorname{Tr}_{R} B,
$$

where the sum is over all representations, including the trivial one. 
The careful reader may note that the operator $(4.28)$ is only equivalent to the usual operator [27, 11]

$$
\exp \sum_{n=1}^{\infty} \frac{e^{-n r_{c}}}{n} \operatorname{Tr} A^{n} \operatorname{Tr} B^{n}
$$

in the $\mathrm{U}(N)$ case. In the more general case where the gauge group is $\mathrm{SO}(N)$ or $\operatorname{Sp}(N)$, the two operators are not equivalent. It turns out that (4.28) is the good operator to use; it would be interesting to investigate further why this is so.

We can now write the total free energy $\mathcal{F}=-\log Z$ (with $Z$ given in (4.25) as

$$
\mathcal{F}=\sum_{i=1}^{3} \mathcal{F}\left(M_{i}\right)+\mathcal{F}_{\text {inst }},
$$

where $\mathcal{F}\left(M_{i}\right)$ are the free energies of the Chern-Simons theories in the spheres $M_{i}, i=$ $1,2,3$, and $\mathcal{F}_{\text {inst }}$ is:

$$
\mathcal{F}_{\text {inst }}=-\ln \left\{\sum_{R_{1}, R_{2}, R_{3}} e^{-\sum_{i=1}^{3} \ell\left(R_{i}\right) r_{c i}} W_{R_{3} R_{1}}\left(\mathcal{L}_{1}\right) W_{R_{1} R_{2}}\left(\mathcal{L}_{2}\right) W_{R_{2} R_{3}}\left(\mathcal{L}_{3}\right)\right\},
$$

where $\mathcal{L}_{i}$ is the link formed by the knots $\left(\Xi_{i}, \Gamma_{i}\right)$ and

$$
\begin{aligned}
W_{R_{3} R_{1}}\left(\mathcal{L}_{1}\right) & =\frac{\left\langle R_{3}\left|V_{M_{1}}\right| R_{1}\right\rangle}{Z_{M_{1}}}, \\
W_{R_{1} R_{2}}\left(\mathcal{L}_{2}\right) & =\frac{\left\langle R_{1}\left|V_{M_{2}}\right| R_{2}\right\rangle}{Z_{M_{2}}}, \\
W_{R_{2} R_{3}}\left(\mathcal{L}_{3}\right) & =\frac{\left\langle R_{2}\left|V_{M_{3}}\right| R_{3}\right\rangle}{Z_{M_{3}}} .
\end{aligned}
$$

It was shown in [1] (using our notation as in figure [) that

$$
V_{M_{1}}=T S^{-1}, \quad V_{M_{2}}=S T^{-1} S, \quad V_{M_{3}}=S^{-1},
$$

which means that the three links $\mathcal{L}_{i}, i=1,2,3$ are Hopf links with linking number +1 and that the framings are as follows: $\left(\Gamma_{1}, \Xi_{3}, \Gamma_{3}\right)$ are canonically framed, i.e. with framings $(0,0,0)$, while $\left(\Xi_{1}, \Xi_{2}, \Gamma_{2}\right)$ have framings $(1,1,1)$, as shown in figure 5 . We can thus write

$$
\begin{aligned}
& W_{R_{3} R_{1}}\left(\mathcal{L}_{1}\right)=(-1)^{\ell\left(R_{3}\right)} q^{\frac{\kappa_{R_{3}}}{2}} \frac{S_{R_{3} R_{1}}^{-1}}{S_{00}}=(-1)^{\ell\left(R_{3}\right)} q^{\frac{\kappa_{R_{3}}}{2}} \mathcal{W}_{R_{3} R_{1}}, \\
& W_{R_{1} R_{2}}\left(\mathcal{L}_{2}\right)=(-1)^{\ell\left(R_{1}\right)+\ell\left(R_{2}\right)} q^{\frac{1}{2}\left(\kappa_{R_{1}}+\kappa_{R_{2}}\right)} \frac{S_{R_{1} R_{2}}^{-1}}{S_{00}}=(-1)^{\ell\left(R_{1}\right)+\ell\left(R_{2}\right)} q^{\frac{1}{2}\left(\kappa_{R_{1}}+\kappa_{R_{2}}\right)} \mathcal{W}_{R_{1} R_{2}} \\
& W_{R_{2} R_{3}}\left(\mathcal{L}_{3}\right)=\frac{S_{R_{2} R_{3}}^{-1}}{S_{00}}=\mathcal{W}_{R_{1} R_{3}},
\end{aligned}
$$

where the $\lambda$ dependent pieces of (4.17) have been absorbed in a redefinition of $r_{c i}$. Therefore (4.31) becomes

$$
\begin{aligned}
\mathcal{F}_{\text {inst }}=-\ln \{1 & +\sum_{R_{1}, R_{2}, R_{3}}(-1)^{\sum_{i=1}^{3} l_{i}} e^{-\sum_{i=1}^{3} \ell\left(R_{i}\right) r_{c i}} q^{\frac{1}{2}\left(\kappa_{R_{1}}+\kappa_{R_{2}}+\kappa_{R_{3}}\right)} \times \\
& \left.\times \mathcal{W}_{R_{3} R_{1}}\left(\mathcal{L}_{1}\right) \mathcal{W}_{R_{1} R_{2}}\left(\mathcal{L}_{2}\right) \mathcal{W}_{R_{2} R_{3}}\left(\mathcal{L}_{3}\right)\right\}
\end{aligned}
$$


where we singled out the term coming from $R_{1}, R_{2}, R_{3}=\cdot$, i.e. the three representations being the trivial representation.

\subsection{Duality map and closed string amplitudes}

Let us first recall the variables we have defined so far. We first defined the Chern-Simons variables $q=e^{g_{s}}$ and $\lambda_{i}=q^{N_{i}+a_{i}}$, with $g_{s}=\frac{2 \pi i}{k_{i}+y}$ being the same for the three theories. We denote the three Kähler parameters of the cylindrical instantons by $r_{c i}, i=1,2,3$ and the three 't Hooft parameters of the different gauge groups by $t_{i}$. We now want to relate the open string parameters $t_{i}$ and $r_{c i}$ to the following closed string parameters: $t$, which is the Kähler parameter of $\mathbb{P}^{2}$, and $s_{i}, i=1,2,3$, which are the Kähler parameters of the two $\mathbb{P}^{1}$ 's and the $\mathbb{R P}^{2}$. The duality map reads

$$
\begin{aligned}
t & =r_{c 1}-\frac{t_{1}+t_{2}}{2}=r_{c 2}-\frac{t_{2}+t_{3}}{2}=r_{c 3}-\frac{t_{1}+t_{3}}{2}, \\
t_{1} & =s_{1}, \quad t_{2}=s_{2}, \quad t_{3}=s_{3} .
\end{aligned}
$$

Let now $q_{i}$ be $q_{i}=e^{-s_{i}}=e^{-t_{i}}, i=1,2,3$, and let $\ell$ be $\ell\left(R_{1}\right)+\ell\left(R_{2}\right)+\ell\left(R_{3}\right)$. We can rewrite the open string partition function (4.35) using (4.36):

$$
\begin{aligned}
\mathcal{F}_{\text {inst }}=-\ln \{1 & +\sum_{\ell}(-1)^{\ell} e^{-\ell t} q^{\frac{1}{2}\left(\kappa_{R_{1}}+\kappa_{R_{2}}+\kappa_{R_{3}}\right)} q_{1}^{\frac{\ell\left(R_{1}\right)+\ell\left(R_{3}\right)}{2}} q_{2}^{\frac{\ell\left(R_{1}\right)+\ell\left(R_{2}\right)}{2}} q_{3}^{\frac{\ell\left(R_{2}\right)+\ell\left(R_{3}\right)}{2}} \times \\
& \left.\times \mathcal{W}_{R_{3} R_{1}}^{\mathrm{U}(N)} \mathcal{W}_{R_{1} R_{2}}^{\mathrm{U}(N)} \mathcal{W}_{R_{2} R_{3}}^{\mathrm{SO}(N) / \mathrm{Sp}(N)}\right\},
\end{aligned}
$$

where the Hopf link invariants in the last line are evaluated at $\lambda=q_{i}^{-1}, i=1,2,3$, respectively. Notice that the leading power of $\lambda$ in $\mathcal{W}_{R_{1} R_{2}}^{\mathrm{U}(N)}$ and in $\mathcal{W}_{R_{1} R_{2}}^{\mathrm{SO}(N) / \mathrm{Sp}(N)}$ is in both cases $\lambda^{\left(\ell\left(R_{1}\right)+\ell\left(R_{2}\right)\right) / 2}$, therefore the above expression for $\mathcal{F}_{\text {inst }}$ gives a power series in $q_{i}$ with positive integer coefficients, as it should. We can now expand the logarithm to find

$$
\mathcal{F}_{\text {inst }}=\sum_{\ell=1}^{\infty} Z_{\ell}^{(c)} e^{-\ell t}
$$

where the connected coefficient $Z_{\ell}^{(c)}$ are given by

$$
Z_{\ell}^{(c)}=\sum_{1 \leq d \leq \ell} \frac{(-1)^{d+1}}{d} \sum_{m_{1}+m_{2}+\cdots+m_{d}=\ell} Z_{m_{1}} Z_{m_{2}} \cdots Z_{m_{d}} .
$$

These coefficients give the instanton partition function order by order in the Kähler parameter $e^{-t}$. Using the formulae given above for Hopf link invariants with classical gauge groups, we can explicitly compute the coefficients $Z_{\ell}^{(c)}$. The contributions independent of the Kähler parameter $t$ are given by the sum of Chern-Simons free energies on $\mathbf{S}^{3}$ $\sum_{i=1}^{3} \mathcal{F}_{C S}\left(M_{i}\right)$, which have already been computed in [3, 1].

\subsection{The oriented contribution}

As we explained in section 2, $\mathcal{F}_{\text {inst }}$ contains contributions due to oriented and to unoriented instantons. In order to compute the closed, unoriented string amplitudes we have to 


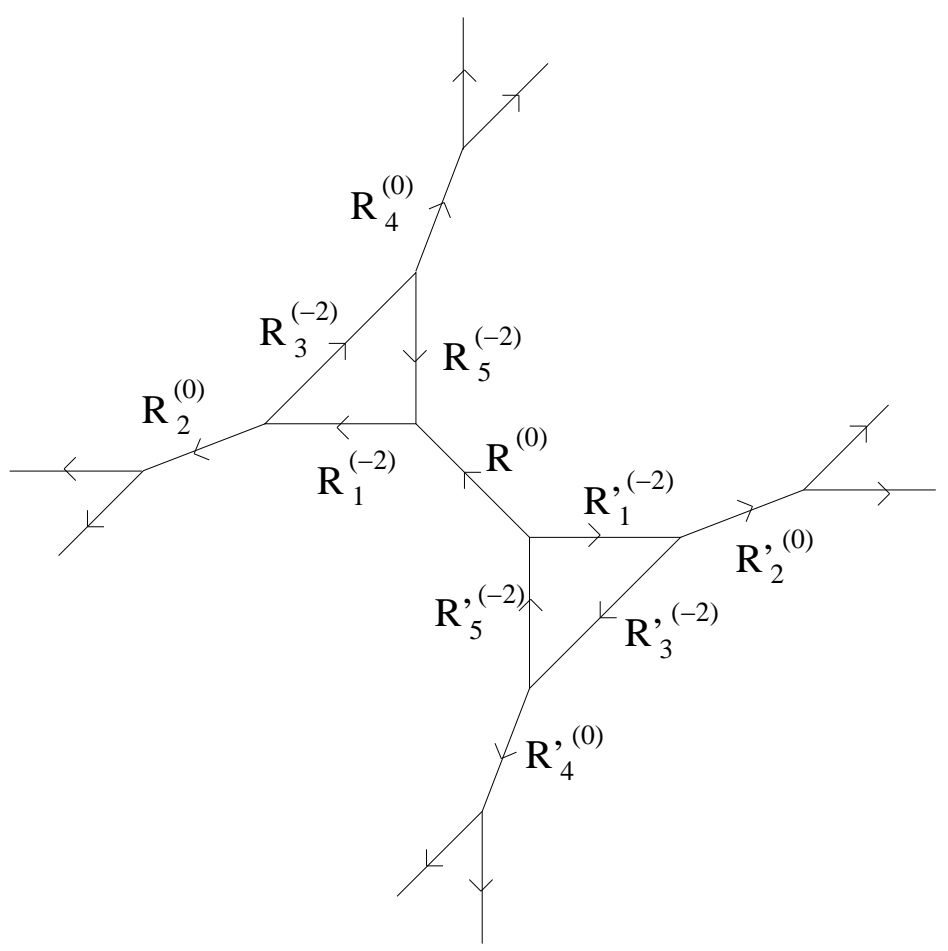

Figure 6: The geometry in the topological vertex formalism. In brackets next to the representations are the framings in the corresponding propagator.

subtract the oriented part, which we must compute independently. The covering space $X$ is the Calabi-Yau manifold depicted in figure 6. The oriented amplitude can be computed using the topological vertex formalism [2. Using the formulas and gluing rules explained in [2] we find

$$
Z(X)=\sum_{R} \mathcal{O}_{R}\left(t, q_{1}, q_{2}\right) \mathcal{O}_{R^{T}}\left(t, q_{1}, q_{2}\right)(-1)^{l(R)} q_{3}^{l(R)}
$$

where $q_{i}=e^{-s_{i}}$ and $t$ is the Kähler parameter of the $\mathbb{P}^{2}, s_{1}$ and $s_{2}$ are the Kähler parameters of the two $\mathbb{P}^{1}$ 's attached to the $\mathbb{P}^{2}$, and $s_{3}$ is the Kähler parameter of the $\mathbb{P}^{1}$ between the two $\mathbb{P}^{2}$ 's. Notice that we have identified the Kähler parameters in the way prescribed by the involution. In (4.40) we introduced the operator

$$
\begin{aligned}
\mathcal{O}_{R}\left(t, q_{1}, q_{2}\right)= & \sum_{R_{i}} C_{R R_{5} R_{1}^{T}} C_{R_{1} R_{3}^{T} R_{2}^{T}} C_{R_{3} R_{5}^{T} R_{4}^{T}} C_{R_{2} . .} C_{R_{4} . .} \times \\
& \times(-1)^{\sum_{i} \ell\left(R_{i}\right)} q^{\kappa\left(R_{1}\right)+\kappa\left(R_{3}\right)+\kappa\left(R_{5}\right)} e^{-\left(\ell\left(R_{1}\right)+\ell\left(R_{3}\right)+\ell\left(R_{5}\right)\right) t} q_{1}^{\ell\left(R_{2}\right)} q_{2}^{\ell\left(R_{4}\right)}
\end{aligned}
$$

where $C_{R_{i} R_{j} R_{k}}$ is the topological vertex amplitude, which can be expressed in terms of the quantities (4.24):

$$
C_{R_{1} R_{2} R_{3}}=\sum_{Q_{1}, Q_{3}, Q} N_{Q Q_{1}}^{R_{1}} N_{Q Q_{3}}^{R_{3}^{T}} q^{\kappa_{R_{2}} / 2+\kappa_{R_{3}} / 2} \frac{W_{R_{2}^{T} Q_{1}} W_{R_{2} Q_{3}}}{W_{R_{2}}} .
$$


Using (4.40) we can express again the free energy as a sum over connected coefficients

$$
\mathcal{F}(X)=-\log Z(X)=\sum_{\ell, \ell_{1}, \ell_{2}, \ell_{3}} Z_{\ell, \ell_{1}, \ell_{2}, \ell_{3}}^{(c)} q_{1}^{\ell_{1}} q_{2}^{\ell_{2}} q_{3}^{\ell_{3}} e^{-\ell t} .
$$

The free energy computed in (4.38) should equal, according to (2.2),

$$
\mathcal{F}_{\text {inst }}=\frac{1}{2} \mathcal{F}(X)+\mathcal{F}\left(\frac{X}{I}, g_{s}\right)_{\text {unor }},
$$

where $\mathcal{F}(X)$ is given in (4.43). This determines the unoriented part, which should have the structure given in (2.5). We will encode the resulting oriented and unoriented GopakumarVafa invariants in the following generating functionals

$$
\begin{aligned}
& \mathcal{F}_{d}^{g, 0}=\frac{1}{2} \sum_{d_{1}, d_{2}, d_{3}} n_{d, d_{1}, d_{2}, d_{3}}^{g, 0} q_{1}^{d_{1}} q_{2}^{d_{2}} q_{3}^{d_{3}}, \\
& \mathcal{F}_{d}^{g, 1}=\sum_{d_{1}, d_{2}, d_{3}} n_{d, d_{1}, d_{2}, d_{3}}^{g, 1} q_{1}^{d_{1}} q_{2}^{d_{2}} q_{3}^{d_{3} / 2}, \\
& \mathcal{F}_{d}^{g, 2}=\sum_{d_{1}, d_{2}, d_{3}} n_{d, d_{1}, d_{2}, d_{3}}^{g, 2} q_{1}^{d_{1}} q_{2}^{d_{2}} q_{3}^{d_{3}},
\end{aligned}
$$

where $d$ is the degree in $e^{-t}$, and the superscripts $g, c$ with $c=0,1,2$ denote the genus and the number of crosscaps, respectively. Of course, $c=0$ is the oriented contribution obtained from (4.43) (multiplied by the factor of $1 / 2$ ), and in the second equation of (4.45) $d_{3}$ must be odd. In order to compute these functionals, we have to remove multicoverings according to (2.3) and (2.5). It is important to note that the requirement that the partition function satisfies the good integrality properties leading to (1.45) is highly nontrivial.

We present the results for the functionals given by (4.45) in appendix B. For the sake of brevity, here we present the results only in the limiting case where we take the volumes of the two $\mathbb{P}^{1}$ 's attached to the $\mathbb{P}^{2}$ to infinity, as in [11]. We thus obtain the answer for the simpler geometry whose toric diagram is depicted in figure 7 This geometry already captures all the interesting features of the unoriented and oriented generating functionals.

To take this limit, one can directly consider the generating functionals (4.45) and set $q_{1,2}=0$, which corresponds to sending the two Kähler parameters to infinity. One can also obtain this limit by taking the leading piece of the $\mathrm{U}(N)$ Hopf link invariants in (4.37), in the sense explained in (4.23). The free energy of this geometry is just:

$$
\mathcal{F}=-\ln \left\{1+\sum_{\ell}(-1)^{\ell} e^{-\ell t} q^{\frac{1}{2}\left(\kappa_{R_{1}}+\kappa_{R_{2}}+\kappa_{R_{3}}\right)} q_{3}^{\frac{\ell\left(R_{2}\right)+\ell\left(R_{3}\right)}{2}} W_{R_{3} R_{1}} W_{R_{1} R_{2}} \mathcal{W}_{R_{2} R_{3}}^{\mathrm{SO}(N) / \operatorname{Sp}(N)}\right\} .
$$

The result can now be encoded in the simpler generating functionals

$$
\mathcal{F}_{d}^{g, 0}=\frac{1}{2} \sum_{d_{3}} n_{d, d_{3}}^{g, 0} q_{3}^{d_{3}}
$$




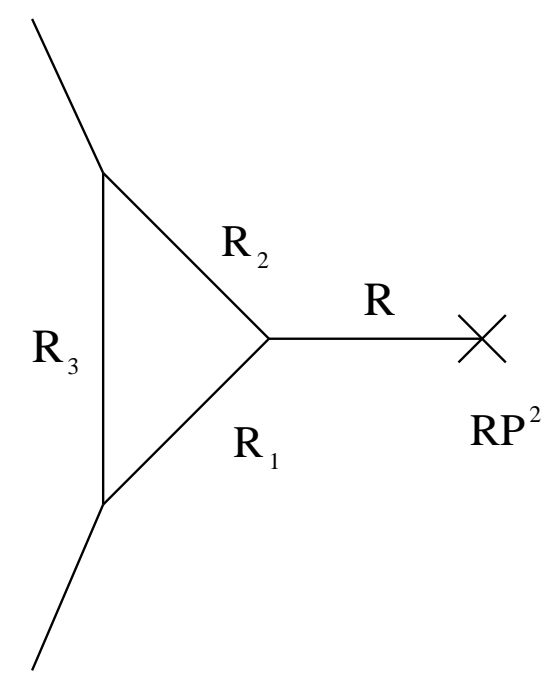

Figure 7: Toric diagram for local $\mathbb{P}^{2}$ attached to an $\mathbb{R P}^{2}$.

\begin{tabular}{|c|c|c|c|c|c|c|c|c|c|c|c|c|c|}
\hline$c=0$ & $d_{3}=0$ & 1 & 2 & 3 & 4 & 5 & $c=1$ & $d_{3}=1$ & 3 & 5 & 7 & 9 & 11 \\
\hline$d=0$ & 0 & 1 & 0 & 0 & 0 & 0 & $d=0$ & 1 & 0 & 0 & 0 & 0 & 0 \\
\hline 1 & 6 & -4 & 0 & 0 & 0 & 0 & 1 & -2 & 0 & 0 & 0 & 0 & 0 \\
\hline 2 & -12 & 14 & -2 & 0 & 0 & 0 & 2 & 5 & -3 & 0 & 0 & 0 & 0 \\
\hline 3 & 54 & -84 & 30 & 0 & 0 & 0 & 3 & -32 & 30 & -4 & 0 & 0 & 0 \\
\hline 4 & -384 & 725 & -392 & 51 & 0 & 0 & 4 & 286 & -369 & 112 & -5 & 0 & 0 \\
\hline 5 & 3390 & -7540 & 5434 & -1368 & 84 & 0 & 5 & -3038 & 5016 & -2410 & 328 & -6 & 0 \\
\hline 6 & -34128 & 87776 & -79198 & 29466 & -4040 & 124 & 6 & 35870 & -72150 & 47554 & -11528 & 819 & -7 \\
\hline
\end{tabular}

Table 1: Invariants $n_{d, d_{3}}^{0, c}$ at genus 0 , with $c=0,1$, up to $d=6$.

\begin{tabular}{|r|rrrr|}
\hline$c=2$ & $d_{3}=2$ & 3 & 4 & 5 \\
\hline$d=3$ & 1 & 0 & 0 & 0 \\
4 & -11 & 2 & 0 & 0 \\
5 & 131 & -66 & 7 & 0 \\
6 & -1690 & 1460 & -333 & 12 \\
\hline
\end{tabular}

Table 2: Invariants $n_{d, d_{3}}^{0,2}$ at genus 0 , up to $d=6$.

$$
\begin{aligned}
\mathcal{F}_{d}^{g, 1} & =\sum_{d_{3}} n_{d, d_{3}}^{g, 1} q_{3}^{d_{3} / 2} \\
\mathcal{F}_{d}^{g, 2} & =\sum_{d_{3}} n_{d, d_{3}}^{g, 2} q_{3}^{d_{3}}
\end{aligned}
$$

with the same restrictions as for (4.45). We present here the all genus results we obtain up to degree 6 in $e^{-t}$. At this order $n_{d, d_{3}}^{g, c}=0 \forall g \geq 11$ (all the invariants $n_{d, d_{3}}^{g, c}$ with $d \leq 6$ that are not shown in the tables are understood to be zero). The results in tables $1-16$ correspond to $\mathrm{Sp}(N)$ gauge group; to obtain the $\mathrm{SO}(N)$ result it suffices to change the sign of the invariants with $c=1$. 


\begin{tabular}{|r|rrrrr|r|rrrrr|}
\hline$c=0$ & $d_{3}=0$ & 1 & 2 & 3 & 4 & $c=1$ & $d_{3}=1$ & 3 & 5 & 7 & 9 \\
\hline$d=3$ & 20 & -18 & 0 & 0 & 0 & $d=3$ & -9 & 7 & 0 & 0 & 0 \\
4 & -462 & 612 & -168 & 0 & 0 & 4 & 288 & -315 & 59 & 0 & 0 \\
5 & 8904 & -15210 & 7380 & -930 & 0 & 5 & -6984 & 9954 & -3630 & 282 & 0 \\
6 & -161896 & 336636 & -228532 & 56536 & -3851 & 6 & 152622 & -269501 & 145467 & -25672 & 1014 \\
\hline
\end{tabular}

Table 3: Invariants $n_{d, d_{3}}^{1, c}$ at genus 1 , with $c=0,1$, up to $d=6$.

\begin{tabular}{|r|rrrr|}
\hline$c=2$ & $d_{3}=2$ & 3 & 4 & 5 \\
\hline$d=4$ & -6 & 0 & 0 & 0 \\
5 & 201 & -55 & 1 & 0 \\
6 & -5180 & 3180 & -438 & 2 \\
\hline
\end{tabular}

Table 4: Invariants $n_{d, d_{3}}^{1,2}$ at genus 1 , up to $d=6$.

\begin{tabular}{|c|c|c|c|c|c|c|c|c|c|c|c|}
\hline$c=0$ & $d_{3}=0$ & 1 & 2 & 3 & 4 & $c=1$ & $d_{3}=1$ & 3 & 5 & 7 & 9 \\
\hline$d=4$ & -204 & 216 & -24 & 0 & 0 & $d=4$ & 108 & -103 & 9 & 0 & 0 \\
\hline 5 & 10860 & -15444 & 5154 & -276 & 0 & 5 & -7506 & 9474 & -2567 & 95 & 0 \\
\hline 6 & -388044 & 690273 & -365536 & 60235 & -1800 & 6 & 329544 & -521400 & 231550 & -29010 & 554 \\
\hline
\end{tabular}

Table 5: Invariants $n_{d, d_{3}}^{2, c}$ at genus 2, with $c=0,1$ up to $d=6$.

\begin{tabular}{|r|rrr|}
\hline$c=2$ & $d_{3}=2$ & 3 & 4 \\
\hline$d=4$ & -1 & 0 & 0 \\
5 & 146 & -18 & 0 \\
6 & -8296 & 3520 & -274 \\
\hline
\end{tabular}

Table 6: Invariants $n_{d, d_{3}}^{2,2}$ at genus 2 , up to $d=6$.

\begin{tabular}{|r|rrrrr|r|rrrrr|}
\hline$c=0$ & $d_{3}=0$ & 1 & 2 & 3 & 4 & $c=1$ & $d_{3}=1$ & 3 & 5 & 7 & 9 \\
\hline$d=4$ & -30 & 28 & 0 & 0 & 0 & $d=4$ & 14 & -12 & 0 & 0 & 0 \\
5 & 7344 & -9094 & 2036 & -30 & 0 & 5 & -4519 & 5133 & -977 & 11 & 0 \\
6 & -581706 & 913220 & -381934 & 40728 & -408 & 6 & 447502 & -642780 & 233460 & -19781 & 139 \\
\hline
\end{tabular}

Table 7: Invariants $n_{d, d_{3}}^{3, c}$ at genus 3 , with $c=0,1$, up to $d=6$.

\begin{tabular}{|r|rrr|}
\hline$c=2$ & $d_{3}=2$ & 3 & 4 \\
\hline$d=5$ & 58 & -2 & 0 \\
6 & -8489 & 2352 & -90 \\
\hline
\end{tabular}

Table 8: Invariants $n_{d, d_{3}}^{3,2}$ at genus 3, up to $d=6$.

\begin{tabular}{|r|rrrrr|r|rrrrr|}
\hline$c=0$ & $d_{3}=0$ & 1 & 2 & 3 & 4 & $c=1$ & $d_{3}=1$ & 3 & 5 & 7 & 9 \\
\hline$d=5$ & 2772 & -3084 & 424 & 0 & 0 & $d=5$ & -1542 & 1599 & -191 & 0 & 0 \\
6 & -580800 & 821490 & -270708 & 17600 & -36 & 6 & 407661 & -536973 & 157255 & -8372 & 13 \\
\hline
\end{tabular}

Table 9: Invariants $n_{d, d_{3}}^{4, c}$ at genus 4 , with $c=0,1$, up to $d=6$. 


\begin{tabular}{|r|rrr|}
\hline$c=2$ & $d_{3}=2$ & 3 & 4 \\
\hline$d=5$ & 12 & 0 & 0 \\
6 & -5862 & 976 & -15 \\
\hline
\end{tabular}

Table 10: Invariants $n_{d, d_{3}}^{4,2}$ at genus 4 , up to $d=6$.

\begin{tabular}{|c|c|c|c|c|c|c|c|c|c|c|c|c|c|}
\hline$c=0$ & $d_{3}=0$ & 1 & 2 & 3 & $c=1$ & $d_{3}=1$ & 3 & 5 & 7 & $c=2$ & $d_{3}=2$ & 3 & 4 \\
\hline$d=5$ & 540 & -552 & 36 & 0 & $d=5$ & -276 & 265 & -15 & 0 & $d=5$ & 1 & 0 & 0 \\
\hline 6 & -393714 & 509724 & -130496 & 4684 & 6 & 254310 & -309962 & 71523 & -2141 & 6 & -2758 & 245 & -1 \\
\hline
\end{tabular}

Table 11: Invariants $n_{d, d_{3}}^{5, c}$ at genus 5 , up to $d=6$.

\begin{tabular}{|r|rrrr|r|rrrr|r|rr|}
\hline$c=0$ & $d_{3}=0$ & 1 & 2 & 3 & $c=1$ & $d_{3}=1$ & 3 & 5 & 7 & $c=2$ & $d_{3}=2$ & 3 \\
\hline$d=5$ & 42 & -40 & 0 & 0 & $d=5$ & -20 & 18 & 0 & 0 & $d=5$ & 0 & 0 \\
6 & -180780 & 216960 & -41904 & 696 & 6 & 108440 & -123342 & 21630 & -302 & 6 & -868 & 34 \\
\hline
\end{tabular}

Table 12: Invariants $n_{d, d_{3}}^{6, c}$ at genus 6 , up to $d=6$.

\begin{tabular}{|c|c|c|c|c|c|c|c|c|c|c|c|}
\hline$c=0$ & $d_{3}=0$ & 1 & & & $c=1$ & $d_{3}=1$ & 3 & 5 & 7 & $c=2$ & $d_{3}=23$ \\
\hline$d=6$ & -55076 & 61896 & -8532 & 244 & $d=6$ & 30948 & -33110 & 4156 & -18 & $d=6$ & -1742 \\
\hline
\end{tabular}

Table 13: Invariants $n_{d, d_{3}}^{7, c}$ at genus 7 , up to $d=6$.

\begin{tabular}{|r|rrr|r|rrr|r|r|}
\hline$c=0$ & $d_{3}=0$ & 1 & 2 & $c=1$ & $d_{3}=1$ & 3 & 5 & $c=2$ & $d_{3}=2$ \\
\hline$d=6$ & -10620 & 11268 & -992 & $d=6$ & 5634 & -5710 & 458 & $d=6$ & -20 \\
\hline
\end{tabular}

Table 14: Invariants $n_{d, d_{3}}^{8, c}$ at genus 8 , up to $d=6$.

\begin{tabular}{|r|rrr|r|rrr|r|r|}
\hline$c=0$ & $d_{3}=0$ & 1 & 2 & $c=1$ & $d_{3}=1$ & 3 & 5 & $c=2$ & $d_{3}=2$ \\
\hline$d=6$ & -1170 & 1180 & -50 & $d=6$ & 590 & -570 & 22 & $d=6$ & -1 \\
\hline
\end{tabular}

Table 15: Invariants $n_{d, d_{3}}^{9, c}$ at genus 9 , up to $d=6$. 


\begin{tabular}{|r|rr|r|rr|}
\hline$c=0$ & $d_{3}=0$ & 1 & $c=1$ & $d_{3}=1$ & 3 \\
\hline$d=6$ & -56 & 54 & $d=6$ & 27 & -25 \\
\hline
\end{tabular}

Table 16: Invariants $n_{d, d_{3}}^{10, c}$ at genus 10, up to $d=6$.

\section{Unoriented localization}

As explained in section 2, to compute the full partition function of closed topological strings on the geometry before the geometric transition, we have to sum both over holomorphic maps from orientable Riemann surfaces to the Calabi-Yau space $X$ as well as maps from non-orientable worldsheets to the orientifolded space $X / I$.

In 泗 it was developed a method for summing unoriented world-sheet instantons for closed topological strings based on localization with respect to a torus action on a moduli space of equivariant holomorphic maps. Although in [4 this moduli space has not been constructed, a computational definition for its virtual fundamental cycle was given. Concretely, this reduces to enumerating all fixed loci under an induced torus action on the moduli space and assigning a local contribution to each component of the fixed locus using an equivariant version of the localization theorem of [28]. Moreover, in [4 it was shown that the fixed loci can be represented in terms of Kontsevich graphs [29] with involution.

This method does not rely on large- $N$ duality, and therefore may provide an independent check of our large- $N$ duality proposal for orientifolds. Namely, we can employ the localization techniques of [⿰氵⿴囗⿱一一] the full closed topological string partition function on the orientifolded geometry before the geometric transition.

We can use the computation in 1 to confirm the one crosscap invariants for low degree and genus obtained from the Chern-Simons computation. There, it was computed the unoriented free energy for a $\mathbb{P}^{2}$ with a $\mathbb{R P}^{2}$ attached. This is exactly the limiting geometry for which we presented our results in tables 1-16, related to the full geometry of section 2 by sending the two Kähler parameters of the two $\mathbb{P}^{1}$ 's of the full geometry to infinity. In our variables, the result of 4 reads

$$
\begin{aligned}
\mathcal{F}= & \frac{1}{g_{s}}\left(q_{3}^{1 / 2}-2 e^{-t} q_{3}^{1 / 2}+5 e^{-2 t} q_{3}^{1 / 2}+\cdots+\frac{1}{9} q_{3}^{3 / 2}-3 e^{-2 t} q_{3}^{3 / 2}+\frac{268}{9} e^{-3 t} q_{3}^{3 / 2}+\cdots\right)+ \\
& +g_{s}\left(\frac{1}{24} q_{3}^{1 / 2}-\frac{1}{12} e^{-t} q_{3}^{1 / 2}+\cdots\right) .
\end{aligned}
$$

By expanding $q=e^{g_{s}}$ in powers of $g_{s}$, it is straightforward to show that the contributions with $c=1$ in tables 116 are in agreement with (2.1).

In the following we will compute some Klein bottle amplitudes using unoriented localization. We will find agreement with the Chern-Simons and with the topological vertex computations presented in the next section. We will perform the computations in the Calabi-Yau geometry $\tilde{X}$. In the patch $\left\{X_{1} \neq 0, X_{7} \neq 0, X_{10} \neq 0\right\}$ we introduce local coordinates

$$
z=\frac{X_{1}^{3} X_{4}}{X_{7} X_{10}^{3}}, \quad u=\frac{X_{6} X_{7} X_{10}^{2}}{X_{1}}, \quad v=\frac{X_{5} X_{7} X_{10}^{2}}{X_{1}}
$$




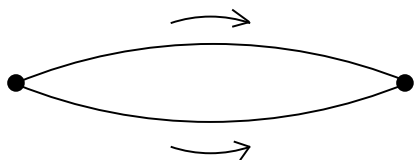

(a)

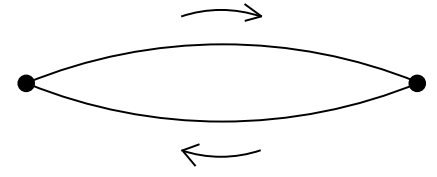

(b)

Figure 8: Two crosscaps and no hyperplane at degree $2 \mathbb{R} \mathbb{P}^{2}$.

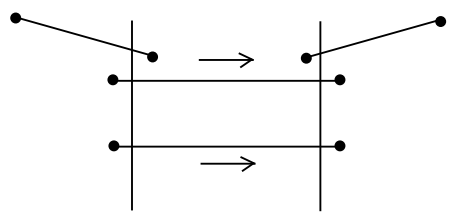

(a)

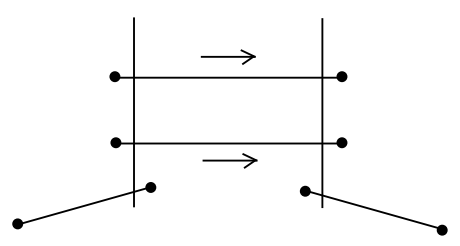

(c)

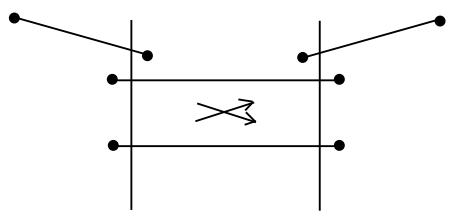

(b)

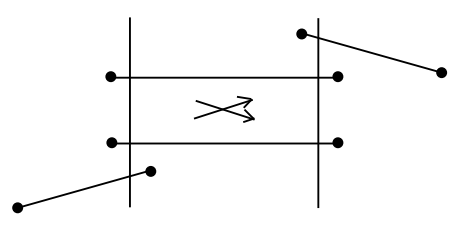

(d)

Figure 9: Two crosscaps and one hyperplane at degree $2 \mathbb{R P}^{2}$. Mirror pairs are $\{(a),(c)\}$ and $\{(b),(d)\}$ respectively.

Using (3.12) we obtain the weights of the local coordinates

$$
\lambda_{z}=6 \lambda_{1}+2 \lambda_{4}, \quad \lambda_{u}=-3 \lambda_{1}-\lambda_{4}+\lambda_{6}, \quad \lambda_{v}=-3 \lambda_{1}-\lambda_{4}+\lambda_{5} .
$$

Note that the compatibility of the involution with the torus action implies $\lambda_{z}+\lambda_{u}+\lambda_{v}=0$.

We will denote the contributions of the fixed loci by $C_{\chi, d, h}$, where $\chi$ is the Euler characteristic of the unoriented source Riemann surface and $d$ and $h$ are the degrees of the map with respect to the $\mathbb{R P}^{2}$ and hyperplane class of $\mathbb{P}^{2}$ respectively.

\subsection{Unoriented localization @ 2 crosscaps @ degree $2 \mathbb{R P}^{2}$}

The computation at degree 0 hyperplane class has been performed in 四 . Let us recall the graphs and their contributions; they are shown in figure 8

Note that in case $(b)$ the antiholomorphic involution exchanges the two components of the source curve. In [ [ it has been postulated that such an operation will introduce an additional minus sign. Therefore the contributions of the two graphs are

$$
C_{0,2,0}^{(a)}=\frac{\lambda_{u} \lambda_{v}}{4 \lambda_{z}^{2}}, \quad C_{0,2,0}^{(b)}=-\frac{\lambda_{u} \lambda_{v}}{4 \lambda_{z}^{2}} .
$$

Let us consider now the degree 1 hyperplane class configurations. The graphs allowed are presented in figure 9 . 


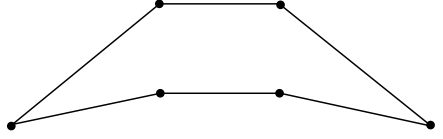

(i)

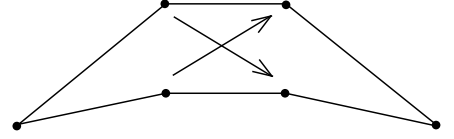

(ii)

Figure 10: Two crosscaps and two hyperplanes at degree $2 \mathbb{R P}^{2}$ : type-II graphs.

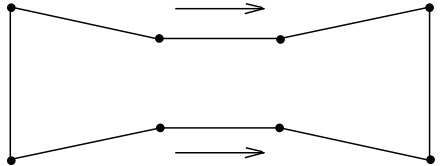

(a)

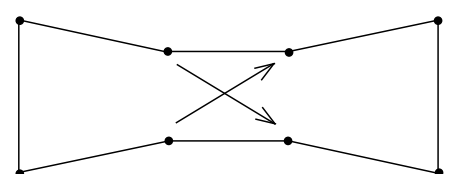

(b)

Figure 11: Two crosscaps and three hyperplanes at degree $2 \mathbb{R P}^{2}$ : type-II graphs.

The allowed configurations are obtained by performing bubblings at the nodes of the graphs in figure 8 and inserting degree 1 hyperplane graphs; we will call such configurations type-I graphs. These come in pairs, each one admits a mirror graph. From now on, we will draw a single graph for each mirror pair. The contributions of the above configurations are given by

$$
C_{0,2,1}^{(a)}=\frac{\lambda_{v}^{2}}{2 \lambda_{z}^{2}}, \quad C_{0,2,1}^{(b)}=-\frac{\lambda_{v}^{2}}{2 \lambda_{z}^{2}}, \quad C_{0,2,1}^{(c)}=\frac{\lambda_{u}^{2}}{2 \lambda_{z}^{2}}, \quad C_{0,2,1}^{(d)}=-\frac{\lambda_{u}^{2}}{2 \lambda_{z}^{2}}
$$

where we have used again the sign rule postulated in 4 . The graph contributions add up to zero.

The discussion is similar at degree 2 hyperplane class. The type-I graphs appearing cancel in pairs due to the same sign rule as above. There also appear new configurations, which we will call type-II graphs, and which we present in figure 10.

Their contributions are given by

$$
C_{0,2,2}^{(i)}=\frac{\left(\lambda_{u}-2 \lambda_{v}\right)\left(\lambda_{v}-\lambda_{u}\right)}{2 \lambda_{v}^{2}}, \quad C_{0,2,2}^{(i i)}=-\frac{\left(\lambda_{u}-2 \lambda_{v}\right)\left(\lambda_{v}-\lambda_{u}\right)}{2 \lambda_{v}^{2}}
$$

and therefore they cancel due to the same sign rule that we used previously.

At degree 3 hyperplane class, we obtain again pairs of graphs of type-I and type-II that cancel each other. In figure 11 we draw some new type-II graphs whose analogues at higher $\mathbb{R P}^{2}$ degree will play an important role.

The contributions of the above two graphs are: $C_{0,2,3}^{(a)}=-C_{0,2,3}^{(b)}=1$. To conclude, we obtain that up to degree 3 hyperplane class, the 2 crosscaps degree $2 \mathbb{R P}^{2}$ Gromov-Witten invariants vanish. In fact, this will be true at any hyperplane class degree.

\subsection{Unoriented localization @ 2 crosscaps @ degree $4 \mathbb{R P}^{2}$}

At degree 0 hyperplane class this computation has been performed in [泪. We list the graphs and their contributions

$$
C_{0,4,0}^{(a)}=\frac{1}{2} \frac{\lambda_{u}^{2} \lambda_{v}^{2}}{\lambda_{z}^{4}}, \quad C_{0,4,0}^{(b)}=-\frac{1}{2} \frac{\lambda_{u}^{2} \lambda_{v}^{2}}{\lambda_{z}^{4}}, \quad C_{0,4,0}^{(c)}=\frac{1}{8} \frac{\lambda_{u} \lambda_{v}\left(2 \lambda_{z}^{2}-9 \lambda_{u} \lambda_{v}\right)}{\lambda_{z}^{4}}
$$




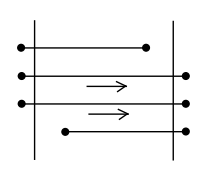

(a)

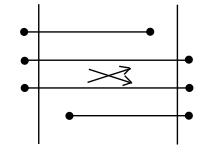

(b)

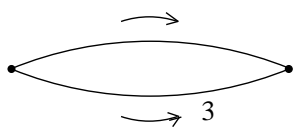

(c)

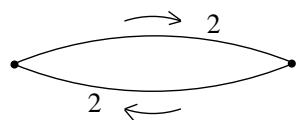

(d)

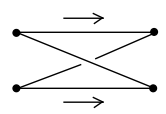

(e)

Figure 12: Two crosscaps and no hyperplane at degree $4 \mathbb{R P}^{2}$.

(A)
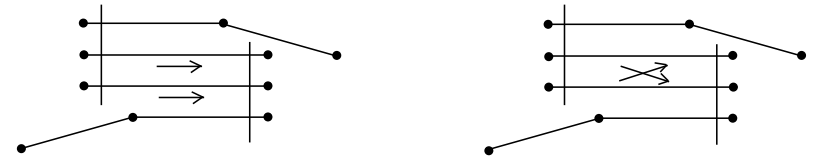

(B)
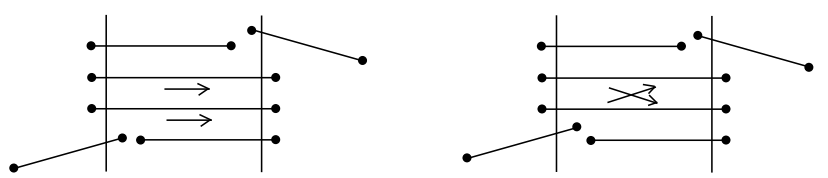

Figure 13: Two crosscaps and one hyperplane at degree $4 \mathbb{R P}^{2}$ : type-III graphs.

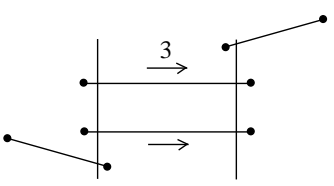

(a)

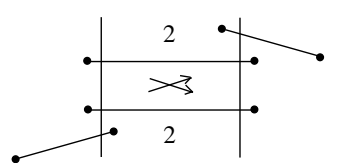

(b)

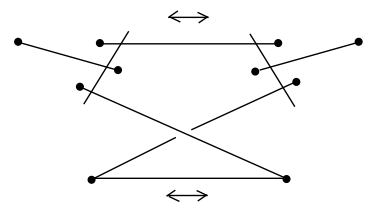

(c)

Figure 14: Two crosscaps and one hyperplane at degree $4 \mathbb{R P}^{2}$ : type-I graphs.

$$
C_{0,4,0}^{(d)}=-\frac{1}{4} \frac{\lambda_{u} \lambda_{v}\left(\lambda_{z}^{2}-4 \lambda_{u} \lambda_{v}\right)}{\lambda_{z}^{4}}, \quad C_{0,4,0}^{(e)}=\frac{1}{8} \frac{\lambda_{u}^{2} \lambda_{v}^{2}}{\lambda_{z}^{4}}
$$

Note that $C_{0,4,0}^{(a)}+C_{0,4,0}^{(b)}=0$ and $C_{0,4,0}^{(c)}+C_{0,4,0}^{(d)}+C_{0,4,0}^{(e)}=0$.

At degree 1 hyperplane class there appear new configurations, which we will call typeIII graphs; they are obtained by adding to the first two graphs in figure 12 degree 1 hyperplane lines as shown in figure 13.

Using again the sign rule in [⿶, the two graphs in each line of figure 13 add up to zero. We now turn to type-I graphs; they are presented in figure 14 and their contributions are:

$$
\begin{aligned}
& C_{0,4,1}^{(a)}=\frac{1}{2}\left(2-9 \frac{\lambda_{u} \lambda_{v}}{\lambda_{z}^{2}}\right)\left(\frac{\lambda_{u}^{2}}{\lambda_{z}^{2}}+\frac{\lambda_{v}^{2}}{\lambda_{z}^{2}}\right), \\
& C_{0,4,1}^{(b)}=\left(-1+4 \frac{\lambda_{u} \lambda_{v}}{\lambda_{z}^{2}}\right)\left(\frac{\lambda_{u}^{2}}{\lambda_{z}^{2}}+\frac{\lambda_{v}^{2}}{\lambda_{z}^{2}}\right), \\
& C_{0,4,1}^{(c)}=\frac{\lambda_{u} \lambda_{v}}{2 \lambda_{z}^{2}}\left(\frac{\lambda_{u}^{2}}{\lambda_{z}^{2}}+\frac{\lambda_{v}^{2}}{\lambda_{z}^{2}}\right) .
\end{aligned}
$$

It is easy to check that $C_{0,4,1}^{(a)}+C_{0,4,1}^{(b)}+C_{0,4,1}^{(c)}=0$. This is in fact the same cancellation that took place at degree 0 hyperplane class between the contributions of the corresponding three graphs. Again, we see that at degree 1 hyperplane class there is nothing essentially new compared to degree 0 hyperplane class. 


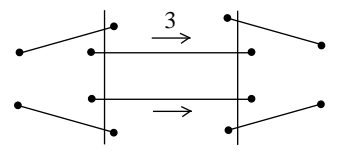

(a)

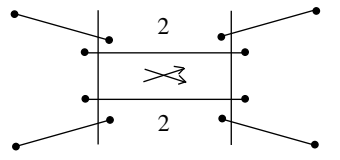

(b)

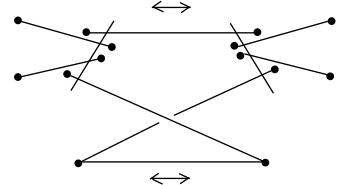

(c)

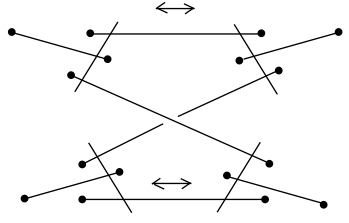

(d)

Figure 15: Two crosscaps and two hyperplanes at degree $4 \mathbb{R} \mathbb{P}^{2}$ : type-I graphs.

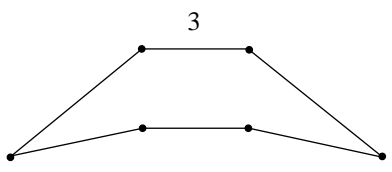

(i)

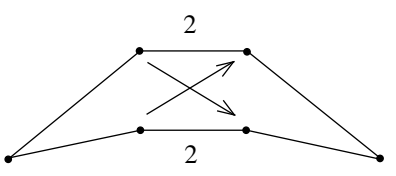

(ii)

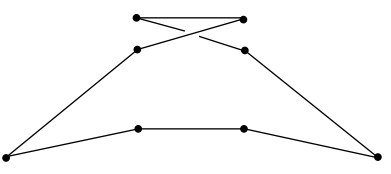

(iii)

Figure 16: Two crosscaps and two hyperplanes at degree $4 \mathbb{R P}^{2}$ : type-II graphs.

Let us now consider the case of degree 2 hyperplane class. We can split the allowed configurations in graphs of type-I and III above. Configurations of type-III are built by starting with the graphs $(a)$ and $(b)$ in figure 12 and further adding in all possible ways degree 2 graphs in $\mathbb{P}^{2}$. They will always cancel in pairs. Configurations of type-I are constructed by starting with the graphs $(c),(d)$ and $(e)$ in figure 12 , performing a bubbling at a pair of identified nodes and inserting degree 2 graphs in $\mathbb{P}^{2}$. The contributions of the graphs with degree 2 multicoverings of one of the hyperplane sections cancel as before; there also appear configurations as in figure 15. However, their contributions also add up to zero, and this will be true for any quartet of type-I graphs as in figure 15.

There are also type-II graphs, which are constructed by starting with the graphs $(c)$, $(d)$ and $(e)$ in figure 12. A triplet of such graphs is presented in figure 16.

The total contribution of these three graphs is

$$
C_{0,4,2}^{(i)}+C_{0,4,2}^{(i i)}+C_{0,4,2}^{(i i i)}=-\frac{\left(\lambda_{u}-\lambda_{v}\right)^{2}\left(\lambda_{u}+\lambda_{v}+\lambda_{z}\right) Q_{9}\left(\lambda_{u}, \lambda_{v}, \lambda_{z}\right)}{4 \lambda_{u}^{2} \lambda_{v}^{2} \lambda_{z}^{2}\left(2 \lambda_{u}+\lambda_{z}\right)^{2}\left(2 \lambda_{v}+\lambda_{z}\right)^{2}\left(3 \lambda_{u}+\lambda_{z}\right)\left(3 \lambda_{v}+\lambda_{z}\right)},
$$

where $Q_{9}\left(\lambda_{u}, \lambda_{v}, \lambda_{z}\right)$ is a degree 9 homogeneous polynomial in $\lambda_{u}, \lambda_{v}, \lambda_{z}$. We recall that consistency of the antiholomorphic involution with the torus action implies $\lambda_{u}+\lambda_{v}+\lambda_{z}=0$, and therefore the sum of the graphs in figure 16 is zero. This will also be true for the other possible triplet of type-II graphs. We conclude that up to degree 2 hyperplane class, the two crosscaps degree $4 \mathbb{R P}^{2}$ Gromov-Witten invariants vanish.

At degree 3 hyperplane class there appear all three types of graphs. We claim that the type-I and III graphs sum up to zero, as above. Besides sets of type-II graphs that have analogues at lower degree hyperplane class, and whose contributions add up to zero in a similar fashion, at degree 3 hyperplane class there also are new collections of graphs. Such a set is presented in figure 17 .

The total contribution of the above three graphs is

$$
C_{0,4,3}^{(i)}+C_{0,4,3}^{(i i)}+C_{0,4,3}^{(i i i)}=\frac{\lambda_{u}\left(\lambda_{u}+\lambda_{v}+\lambda_{z}\right)\left(\lambda_{u}-\lambda_{v}\right)^{2}\left(\lambda_{u}-2 \lambda_{v}\right)^{2} Q_{3}\left(\lambda_{u}, \lambda_{v}, \lambda_{z}\right)}{2 \lambda_{z}^{2} \lambda_{v}^{4}\left(2 \lambda_{v}+\lambda_{z}\right)^{2}\left(3 \lambda_{v}+\lambda_{z}\right)},
$$




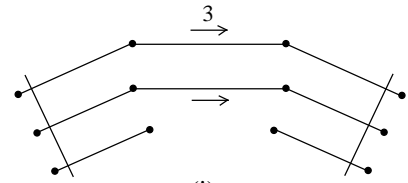

(i)

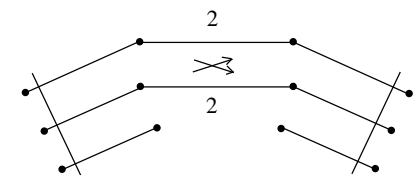

(ii)

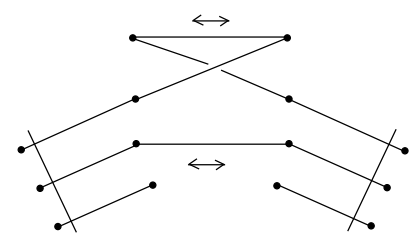

(iii)

Figure 17: Two crosscaps and three hyperplanes at degree $4 \mathbb{R P}^{2}$ : type-II graphs.

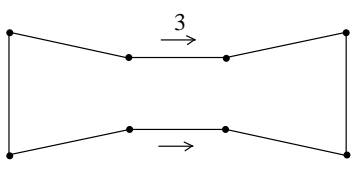

(a)

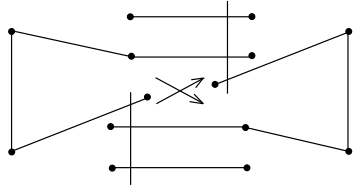

(d)

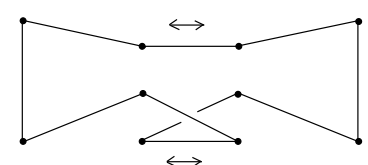

(b)

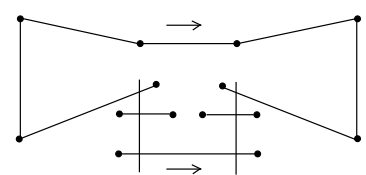

(e)

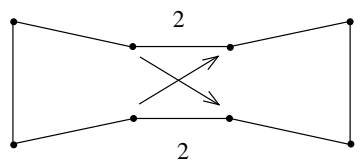

(c)

Figure 18: Two crosscaps and three hyperplanes at degree $4 \mathbb{R P}^{2}$ : type-II graphs.

where $Q_{3}\left(\lambda_{u}, \lambda_{v}, \lambda_{z}\right)$ is a degree 3 homogeneous polynomial in $\lambda_{u}, \lambda_{v}, \lambda_{z}$. But $\lambda_{u}+\lambda_{v}+\lambda_{z}=$ 0 , and these graphs sum up to zero.

However, at degree 3 hyperplane class there is a unique set of type-II graphs, presented in figure 18, whose total contribution does not vanish.

The contributions of the graphs in figure 18 are given by

$$
\begin{aligned}
& C_{0,4,3}^{(a)}=1-3 \frac{\lambda_{u} \lambda_{v}}{\lambda_{z}^{2}}, \quad C_{0,4,3}^{(b)}=\frac{\lambda_{u} \lambda_{v}}{\lambda_{z}^{2}}, \quad C_{0,4,3}^{(c)}=2 \frac{\lambda_{u} \lambda_{v}}{\lambda_{z}^{2}}, \\
& C_{0,4,3}^{(d)}=-1+2 \frac{\lambda_{u} \lambda_{v}}{\lambda_{z}^{2}}, \quad C_{0,4,3}^{(e)}=1-2 \frac{\lambda_{u} \lambda_{v}}{\lambda_{z}^{2}} .
\end{aligned}
$$

We see that the sum of the above expressions is equal to 1, which is the Gromov-Witten invariant $n_{3,2}^{0,2}$ of table 1. It is straightforward to perform now a similar computation but taking also into account the two $(-1,-1)$ curves that are transversal to the $\mathbb{P}^{2}$. The result is that at degree 3 hyperplane class we obtain the following contribution to the free energy from 2 crosscap configurations

$$
\mathcal{F}_{3}^{0,2}=q_{3}^{2}-q_{1} q_{3}^{2}-q_{2} q_{3}^{2}+q_{1} q_{2} q_{3}^{2} .
$$

This is in agreement with the Chern-Simons theory result presented in appendix B.

\section{Topological vertex computation}

Using large- $N$ duality, it was recently proposed [2] that the free energy of closed topological strings on a toric manifold can be computed using a cubic field theory, namely a 
topological vertex and gluing rules. In this section we present a prescription to compute all genus topological string amplitudes on orientifolds with an external " $\mathbb{R} \mathbb{P}^{2}$ leg" by using the topological vertex formalism. We will also explicitly show that this prescription is equivalent to the large- $N$ dual Chern-Simons computation.

\subsection{General prescription}

Consider a quotient $X / I$ of a local, toric Calabi-Yau manifold $X$ by an involution $I$ which can be represented as in figure 19. We have a bulk geometry, represented by the blob, attached to an $\mathbb{R P}^{2}$ through an edge associated to the representation $R$. Let us denote by $\mathcal{O}_{R}$ the amplitude for the blob with the external leg. We propose the following formula for total partition function:

$$
Z=\sum_{R=R^{T}} \mathcal{O}_{R} Q^{\ell(R) / 2}(-1)^{\frac{1}{2}(\ell(R) \mp r(R))}
$$

where the sum is over all self-conjugate representations $R$. Here $r(R)$ denotes the rank of $R$, as in (4.12), and $Q=e^{-s}$ is the exponentiated Kähler parameter corresponding to the $\mathbb{R P}^{2}$. The $\mp$ sign is correlated with the choice of \pm sign for the crosscaps, and corresponds to the SO / Sp gauge duals, respectively.

The prescription (6.1) comes from the action of the involution $I$ on the partition function on the covering space, which is given by

$$
Z=\sum_{R} \mathcal{O}_{R}\left(t_{i}\right) \mathcal{O}_{R^{T}}\left(t_{i}\right) Q^{\ell(R)}(-1)^{\ell(R)}
$$

where the Kähler parameters have been identified in the way prescribed by the involution. The involution $I$ maps one half of the toric diagram onto the other half, reversing the orientation of the middle leg. The resulting partition function is the one given by (6.1).

We are presently investigating in more details the origin of (6.1). Having a clear understanding of this formula will probably allow us to define a similar prescription for involutions with a fixed locus, like the $I^{+}$of $[8]$.

The restriction to self-conjugate representations may appear surprising at first sight. But in the topological vertex formalism, inverting the orientation of one edge sends $R$ to its transpose $R^{T}$ (and also introduces a factor of $(-1)^{\ell(R)}$ ). Therefore, since the $\mathbb{R} \mathbb{P}^{2}$ leg is unoriented, its partition function must sum only over self-conjugate representations, which are the only representations consistent with the involution $I$.

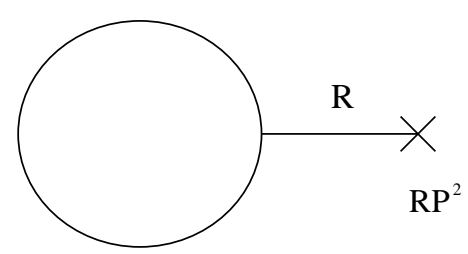

Figure 19: Toric diagram for the quotient $X / I$ of a local, toric Calabi-Yau manifold with a single $\mathbb{R P}^{2}$. 
It is interesting to note that the formula (6.1) is very similar to the formula for quantum dimensions of SO/Sp gauge group in terms of $\mathrm{U}(N)$ quantum dimensions (4.12). Both formulas share the constraint $R=R^{T}$ and the factor of $(-1)^{\frac{1}{2}(\ell(R) \mp r(R))}$. This gives a geometrical argument, from the topological vertex formalism, for the appearance of $\mathrm{SO} / \mathrm{Sp}$ gauge groups on the Chern-Simons side.

\subsection{Examples}

We now consider two examples of the above prescription.

1) Orientifold of the resolved conifold. The simplest example is the orientifold of the resolved conifold first considered in [3], which we reviewed in section 3 In that case, the toric diagram is very simple and has been drawn in the left hand side of figure 1 . The rule (6.1) gives:

$$
F=-\log \left\{\sum_{R=R^{T}} C_{. . R} Q^{\ell(R) / 2}(-1)^{\frac{1}{2}(\ell(R) \mp r(R))}\right\} .
$$

This should equal the free energy of Chern-Simons on the sphere for the gauge groups SO / Sp (3.2). The free energy (6.3) can be indeed computed exactly by using the following key formula due to Littlewood [19, 30]:

$$
\sum_{R=R^{T}} s_{R}\left(x_{i}\right)(-1)^{\frac{1}{2}(\ell(R) \mp r(R))}=\prod_{i=1}^{\infty}\left(1 \pm x_{i}\right) \prod_{1 \leq i<j<\infty}\left(1-x_{i} x_{j}\right) .
$$

Since $C_{R}$. $=W_{R}=s_{R}\left(q^{-i+\frac{1}{2}}\right)$, we can compute (6.3) by setting $x_{i}=q^{-i+\frac{1}{2}} Q^{\frac{1}{2}}$ in the r.h.s. of (6.4). First of all, notice that

$$
\prod_{i, j}\left(1-q^{-i-j+1} Q\right)=\exp \left\{-\sum_{n=1}^{\infty} \frac{Q^{n}}{n\left(q^{\frac{n}{2}}-q^{-\frac{n}{2}}\right)^{2}}\right\} .
$$

Also, we can easily compute that

$$
\prod_{i}\left(1 \mp q^{-i+\frac{1}{2}} Q^{\frac{1}{2}}\right)=\exp \left\{-\sum_{n=1}^{\infty} \frac{( \pm 1)^{n} Q^{\frac{n}{2}}}{n\left(q^{\frac{n}{2}}-q^{-\frac{n}{2}}\right)}\right\}
$$

and from this we easily check that, indeed, the free energy computed in (6.3) equals (3.2).

2) Local $\mathbb{P}^{2}$ attached to $\mathbb{R P}^{2}$. The second example to consider is local $\mathbb{P}^{2}$ attached to a single $\mathbb{R P}^{2}$, whose toric diagram is drawn in figure ? and which was discussed before from the point of view of geometric transitions. The amplitude for this geometry is given by (6.1) with

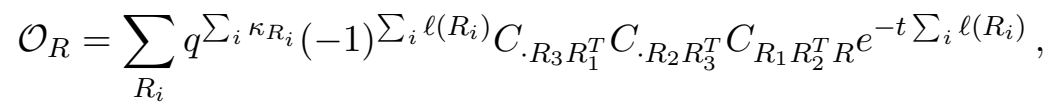

and $t$ is the Kähler parameter of local $\mathbb{P}^{2}$. If we now compare this expression to the one obtained by geometric transition in (4.46), we find that both amplitudes are equal if

$$
\frac{1}{S_{00}^{\mathrm{SO}(N) / \mathrm{Sp}(N)}} \sum_{R=R^{T}} C_{R_{1} R_{2}^{T} R} Q^{\ell(R) / 2}(-1)^{\frac{1}{2}(\ell(R) \mp r(R))}=q^{-\frac{\kappa_{R_{2}}}{2}} Q^{\frac{1}{2}\left(\ell\left(R_{1}\right)+\ell\left(R_{2}\right)\right)} \mathcal{W}_{R_{1} R_{2}}^{\mathrm{SO}(N) / \mathrm{Sp}(N)},
$$


where we have taken into account that the partition function of the geometry in figure 7 also includes a $t$-independent piece which equals $S_{00}^{\mathrm{SO}(N) / \mathrm{Sp}(N)}$. The r.h.s. of (6.8) involves the Hopf link invariant for the gauge groups SO / Sp, where we put $\lambda=Q^{-1}$. Notice that $Q^{\frac{1}{2}\left(\ell\left(R_{1}\right)+\ell\left(R_{2}\right)\right)} \mathcal{W}_{R_{1} R_{2}}^{\mathrm{SO}(N) / \mathrm{Sp}(N)}$ is a polynomial in $Q^{\frac{1}{2}}$, while the l.h.s. of (6.8) is a priori an infinite series in $Q^{\frac{1}{2}}$. The identity (6.8) can be easily proven in the simple case that $R_{1}$ (or $R_{2}$ ) is the trivial representation, by using again the key identity (6.4). Let us sketch the proof in the $S p$ case, the $S O$ case being similar.

First, notice that, as we have just shown in the example of the orientifold of the conifold, $S_{00}^{\mathrm{SO}(N) / \mathrm{Sp}(N)}$ equals the l.h.s. of (6.4) evaluated at $x_{i}=q^{-i+\frac{1}{2}} Q^{\frac{1}{2}}$. Let us consider the l.h.s. of (6.4) for the $+\operatorname{sign}\left(\right.$ i.e. the - sign in the r.h.s.), but now evaluated at $x_{i}=q^{\mu_{i}-i+\frac{1}{2}} Q^{\frac{1}{2}}$, where $\mu=\left\{\mu_{i}\right\}_{i=1, \ldots, d(\mu)}$ is the partition corresponding to the representation $R_{1}^{T}$. After dividing it by $S_{00}^{\mathrm{Sp}(N)}$ we find a finite product

$$
\prod_{i=1}^{d(\mu)} \frac{1-q^{\mu_{i}-i+\frac{1}{2}} Q^{\frac{1}{2}}}{1-q^{-i+\frac{1}{2}} Q^{\frac{1}{2}}} \prod_{1 \leq i<j \leq d(\mu)} \frac{1-q^{\mu_{i}+\mu_{j}-i-j+1} Q^{\frac{1}{2}}}{1-q^{-i-j+1} Q^{\frac{1}{2}}} \prod_{i=1}^{d(\mu)} \prod_{v=1}^{\mu_{i}}\left(1-q^{\mu_{i}-i-d(\mu)-v+1}\right) .
$$

After some massaging, and using the explicit formulae (4.7), (4.9), as well as the relation (4.11), it is easy to see that (6.9) equals

$$
Q^{\frac{\ell(\mu)}{2}} \frac{\mathcal{W}_{R_{1}}^{\mathrm{Sp}(N)}\left(\lambda=Q^{-1}\right)}{W_{R_{1}}}
$$

where $W_{R}=s_{R}\left(q^{-i+\frac{1}{2}}\right)$. Using now that $C_{R R_{1}}$. $=W_{R R_{1}^{T}} q^{\kappa_{R_{1}} / 2}$ as well as the explicit formula (4.24), we see that indeed (6.8) is satisfied when $R_{2}=$. in the case of $\operatorname{Sp}(N)$.

Although we don't have a full proof of (5.8) in general, we have checked it in many cases. This shows indeed that the topological vertex calculation and the geometric transition computation give the same result for this geometry, and indeed for all the geometries of the form depicted in figure 19 .

\section{Discussion and open problems}

In this paper we have seen how to compute topological string amplitudes on a certain class of Calabi-Yau orientifolds, by using geometric transitions involving SO / Sp Chern-Simons theory, the topological vertex formalism, and localization techniques. This allows us to extract BPS invariants counting higher genus curves with one and two crosscaps.

This work can be extended in various ways. First of all, it would be very interesting to consider Calabi-Yau orientifolds in which the involution has fixed loci, like for example the ones considered in [8]. In this case, the geometric transition of [3] is no longer useful and one has to find other ways of implementing a Chern-Simons dual description. In the context of the topological vertex formalism, we should find the right prescription to deal with fixed point loci, by using perhaps the group-theoretic results of [19] for SO/Sp. Secondly, one should consider open string amplitudes by adding lagrangian D-branes, and to clarify in this way the BPS content of SO / Sp Chern-Simons invariants of knots and links. 
It would be also very important to clarify some issues that appeared in the orientifolds that we studied here. For example, one would like to have a more detailed derivation of the multicovering formulae for amplitudes involving two crosscaps and of the choice of annulus operator we made, as well as a more rigorous justification of the localization techniques we used. We expect to report on these problems in future work.

\section{Acknowledgments}

We would like to thank S. Kadir for collaboration at the initial stages of this project and I. Antoniadis, D.-E. Diaconescu and N. Wyllard for useful conversations. The work of V.B. was supported by a Rhodes scholarship. The work of B.F. was supported in part by DOE grant DE-FG02-96ER40959.

\section{A. Subsets of Young tableaux}

To compute the tensor product decomposition of irreducible representations of $\mathrm{SO}(N)$ and $\operatorname{Sp}(N)$ using Littlewood's technique as explained in (4.20), we had to use four different subsets of Young tableaux: $\{\delta\}$ and $\{\gamma\}$ for $\operatorname{SO}(N)$, and $\{\beta\}$ and $\{\alpha\}$ for $\operatorname{Sp}(N)$. These four sets are defined as follows [19].

$\{\delta\}$ is the set of all partitions into even parts only: $\{$ Ш, س, 田, .. $\}$.

$\{\beta\}$ is the set of all partitions such that there are an even number of parts of any given magnitude: $\{$ 日, 田, 目, ...

To define the two remaining sets we have to use the Frobenius notation [19, 18. In this notation, a Young tableau is described by an array of pair of numbers. The number of pairs is equal to the number of boxes in the leading diagonal of the tableau; the upper number of the pair is the number of boxes to the right and the lower number is the number of boxes below. For example, the Young tableau $\mathbb{\boxplus}$ is described in the Frobenius notation by $\left(\begin{array}{ll}2 & 1 \\ 2 & 0\end{array}\right)$.

Using this notation we can define the two remaining sets. Consider Young tableaux defined in the Frobenius notation by

$$
\left(\begin{array}{lllll}
a_{1} & a_{2} & a_{3} & a_{4} & \ldots \\
b_{1} & b_{2} & b_{3} & b_{4} & \ldots
\end{array}\right)
$$

$\{\gamma\}$ is the set of Young tableaux such that $a_{i}=b_{i}+1 \forall i$ :

$\{\alpha\}$ is the set of Young tableaux such that $a_{i}+1=b_{i} \forall i$ : \{日, 田, 目, ...

Note that $\{\beta\}$ and $\{\alpha\}$ are respectively related to $\{\delta\}$ and $\{\gamma\}$ by taking the transpose of the representations, where by transpose we mean exchanging rows and columns.

\section{B. Results in the general case}

Here we present the results for the full generating functionals given by (4.45). The \pm sign corresponds to $S p$ and $S O$, respectively. Of course, the oriented contribution for $q_{3}=0$ 
agrees with previous results for the local del Pezzo $d P_{3}$ with one Kähler parameter sent to infinity [31, 11], and if we set $q_{1,2}=0$ we recover the results presented in tables 1 1 [16 (taking into account the $1 / 2$ factor in the definition of the $c=0$ generating functional).

We computed the results up to degree 5 in $e^{-t}$, but we will present only the results up to degree 3 as the higher degree results are rather cumbersome.

$$
\begin{aligned}
& \mathcal{F}_{0}^{0,0}=q_{1}+q_{2}+\frac{1}{2} q_{3}, \\
& \mathcal{F}_{0}^{0,1}= \pm\left[q_{3}^{1 / 2}\right] \\
& \mathcal{F}_{0}^{0,2}=0 \text {, } \\
& \mathcal{F}_{1}^{0,0}=3-2\left(q_{1}+q_{2}+q_{3}\right)+\left(q_{1} q_{2}+q_{2} q_{3}+q_{1} q_{3}\right), \\
& \mathcal{F}_{1}^{0,1}= \pm\left[-2 q_{3}^{1 / 2}+\left(q_{1} q_{3}^{1 / 2}+q_{2} q_{3}^{1 / 2}\right)\right] \\
& \mathcal{F}_{1}^{0,2}=0 \text {, } \\
& \mathcal{F}_{2}^{0,0}=-6+5\left(q_{1}+q_{2}\right)+7 q_{3}-4 q_{1} q_{2}-6\left(q_{1} q_{3}+q_{2} q_{3}\right)+4 q_{1} q_{2} q_{3}+\frac{1}{2}\left(q_{1}^{2} q_{3}+q_{2}^{2} q_{3}\right)- \\
& -q_{3}^{2}+\left(q_{1} q_{3}^{2}+q_{2} q_{3}^{2}\right)-q_{1} q_{2} q_{3}^{2} \\
& \mathcal{F}_{2}^{0,1}= \pm\left[5 q_{3}^{1 / 2}-4\left(q_{1} q_{3}^{1 / 2}+q_{2} q_{3}^{1 / 2}\right)+3 q_{1} q_{2} q_{3}^{1 / 2}-3 q_{3}^{3 / 2}+2\left(q_{1} q_{3}^{3 / 2}+q_{2} q_{3}^{3 / 2}\right)-q_{1} q_{2} q_{3}^{3 / 2}\right] \text {, } \\
& \mathcal{F}_{2}^{0,2}=0 \text {, } \\
& \mathcal{F}_{3}^{0,0}=27-32\left(q_{1}+q_{2}\right)-42 q_{3}+35 q_{1} q_{2}+48\left(q_{1} q_{3}+q_{2} q_{3}\right)-50 q_{1} q_{2} q_{3}+7\left(q_{1}^{2}+q_{2}^{2}\right)+ \\
& +15 q_{3}^{2}-6\left(q_{1}^{2} q_{2}+q_{1} q_{2}^{2}\right)-10\left(q_{1}^{2} q_{3}+q_{2}^{2} q_{3}\right)-16\left(q_{1} q_{3}^{2}+q_{2} q_{3}^{2}\right)+8\left(q_{1}^{2} q_{2} q_{3}+q_{1} q_{2}^{2} q_{3}\right)+ \\
& +3\left(q_{1}^{2} q_{3}^{2}+q_{2}^{2} q_{3}^{2}\right)-2\left(q_{1}^{2} q_{2} q_{3}^{2}+q_{1} q_{2}^{2} q_{3}^{2}\right)+15 q_{1} q_{2} q_{3}^{2}, \\
& \mathcal{F}_{3}^{0,1}= \pm\left[-32 q_{3}^{1 / 2}+35\left(q_{1} q_{3}^{1 / 2}+q_{2} q_{3}^{1 / 2}\right)-36 q_{1} q_{2} q_{3}^{1 / 2}-6\left(q_{1}^{2} q_{3}^{1 / 2}+q_{2}^{2} q_{3}^{1 / 2}\right)+\right. \\
& +\left(q_{1}^{2} q_{2} q_{3}^{1 / 2}+q_{1} q_{2}^{2} q_{3}^{1 / 2}\right)+30 q_{3}^{3 / 2}-30\left(q_{1} q_{3}^{3 / 2}+q_{2} q_{3}^{3 / 2}\right)+4\left(q_{1}^{2} q_{3}^{3 / 2}+q_{2}^{2} q_{3}^{3 / 2}\right)+ \\
& \left.+28 q_{1} q_{2} q_{3}^{3 / 2}-3\left(q_{1}^{2} q_{2} q_{3}^{3 / 2}+q_{1} q_{2}^{2} q_{3}^{3 / 2}\right)-4 q_{3}^{5 / 2}+3\left(q_{1} q_{3}^{5 / 2}+q_{2} q_{3}^{5 / 2}\right)-2 q_{1} q_{2} q_{3}^{5 / 2}\right], \\
& \mathcal{F}_{3}^{0,2}=q_{3}^{2}-\left(q_{1} q_{3}^{2}+q_{2} q_{3}^{2}\right)+q_{1} q_{2} q_{3}^{2}, \\
& \mathcal{F}_{3}^{1,0}=10-9\left(q_{1}+q_{2}+q_{3}\right)+8\left(q_{1} q_{2}+q_{1} q_{3}+q_{2} q_{3}\right)-7 q_{1} q_{2} q_{3}, \\
& \mathcal{F}_{3}^{1,1}= \pm\left[-9 q_{3}^{1 / 2}+8\left(q_{1} q_{3}^{1 / 2}+q_{2} q_{3}^{1 / 2}\right)-7 q_{1} q_{2} q_{3}^{1 / 2}+7 q_{3}^{3 / 2}-\right. \\
& \left.-6\left(q_{1} q_{3}^{3 / 2}+q_{2} q_{3}^{3 / 2}\right)+5 q_{1} q_{2} q_{3}^{3 / 2}\right] \\
& \mathcal{F}_{3}^{1,2}=0 \text {. }
\end{aligned}
$$

\section{References}

[1] R. Gopakumar and C. Vafa, On the gauge theory/geometry correspondence, Adv. Theor. Math. Phys. 3 (1999) 1415 hep-th/9811131.

[2] M. Aganagic, A. Klemm, M. Marino and C. Vafa, The topological vertex, hep-th/0305132,

[3] S. Sinha and C. Vafa, SO and Sp Chern-Simons at large- $N$, hep-th/0012136.

[4] D.-E. Diaconescu, B. Florea and A. Misra, Orientifolds, unoriented instantons and localization, J. High Energy Phys. 07 (2003) 041 hep-th/0305021.

[5] K. Hori et al., Mirror symmetry, C. Vafa and E. Zaslow eds., American Mathematical Society, Brookline 2003. 
[6] M. Bershadsky, S. Cecotti, H. Ooguri and C. Vafa, Kodaira-Spencer theory of gravity and exact results for quantum string amplitudes, Commun. Math. Phys. 165 (1994) 311 hep-th/9309140.

[7] I. Antoniadis, E. Gava, K.S. Narain and T.R. Taylor, Topological amplitudes in string theory, Nucl. Phys. B 413 (1994) 162 hep-th/9307158.

[8] B. Acharya, M. Aganagic, K. Hori and C. Vafa, Orientifolds, mirror symmetry and superpotentials, hep-th/0202208.

[9] I. Brunner and K. Hori, Orientifolds and mirror symmetry, J. High Energy Phys. 11 (2004) 005 hep-th/0303135.

[10] R. Gopakumar and C. Vafa, M-theory and topological strings, II, hep-th/9812127.

[11] M. Aganagic, M. Marino and C. Vafa, All loop topological string amplitudes from Chern-Simons theory, Commun. Math. Phys. 247 (2004) 467 hep-th/0206164.

[12] M. Aganagic and C. Vafa, $G_{2}$ manifolds, mirror symmetry and geometric engineering, hep-th/0110171.

[13] D.-E. Diaconescu, B. Florea and A. Grassi, Geometric transitions and open string instantons, Adv. Theor. Math. Phys. 6 (2003) 619 hep-th/0205234.

[14] D.-E. Diaconescu, B. Florea and A. Grassi, Geometric transitions, del Pezzo surfaces and open string instantons, Adv. Theor. Math. Phys. 6 (2003) 643 hep-th/0206163.

[15] E. Witten, Quantum field theory and the Jones polynomial, Commun. Math. Phys. 121 (1989) 351 .

[16] M. Marino and C. Vafa, Framed knots at large- $N$, hep-th/0108064.

[17] P. Cvitanovic', Group theory, http://www.cns.gatech.edu/ predrag

[18] S. Ramgoolam, Comment on two-dimensional $O(N)$ and $\operatorname{Sp}(N)$ Yang-Mills theories as string theories, Nucl. Phys. B 418 (1994) 30 hep-th/9307085.

[19] D.E. Littlewood, The theory of group characters and matrix representations of groups, The Clarendon Press, Oxford 1940.

[20] M. Aganagic, A. Klemm and C. Vafa, Disk instantons, mirror symmetry and the duality web, Z. Naturforsch. A57 (2002) 1-28 hep-th/0105045].

[21] E. Guadagnini, The universal link polynomial, [nt. J. Mod. Phys. A 7 (1992) 877.

[22] R.C. King, Modification rules and products of irreducible representations of the unitary, orthogonal, and symplectic groups, J. Math. Phys. 12 (1971) 1588.

[23] W. Fulton and J. Harris, Representation theory: a first course, Springer Verlag, Berlin 1997.

[24] A. Okounkov, N. Reshetikhin and C. Vafa, Quantum Calabi-Yau and classical crystals, hep-th/0309208.

[25] T. Eguchi and H. Kanno, Geometric transitions, Chern-Simons gauge theory and Veneziano type amplitudes, Phys. Lett. B 585 (2004) 163 hep-th/0312234.

[26] E. Witten, Chern-Simons gauge theory as a string theory, Prog. Math. 133 (1995) 637-678 hep-th/9207094. 
[27] H. Ooguri and C. Vafa, Knot invariants and topological strings, Nucl. Phys. B 577 (2000) 419 hep-th/9912123.

[28] T. Graber and R. Pandharipande, Localization of virtual classes, Invent. Math. 135 (1999) 487 .

[29] M. Kontsevich, Enumeration of rational curves via torus actions, hep-th/9405035.

[30] I.G. Macdonald, Symmetric functions and Hall polynomials, Oxford University Press, Oxford 1995.

[31] T.M. Chiang, A. Klemm, S.T. Yau and E. Zaslow, Local mirror symmetry: calculations and interpretations, Adv. Theor. Math. Phys. 3 (1999) 495 hep-th/9903053. 